\title{
THERMAL-INDUCED DIMERIZATION CYCLIZATION OF ETHYL N-(STYRYLCARBAMOYL)ACETATES: FORMATION OF 4-HYDROXY-2(1H)-PYRIDONE-3-CARBOXAMIDE DERIVATIVES
}

\author{
Sheng-Yin Zhao", Bao-Shuo Liu, Jing Huang, Shao-Hua Cheng, Yun-xia Deng \\ and Zhi-Yu Shao
}

Department of Chemistry, Donghua University, Shanghai 201620, P. R. China, Tel: +8621 67792436; Fax: +86 21 67792608-805; E-mail:syzhao8@dhu.edu.cn

Table of contents

General information

Synthesis and spectral data of (E)-diethyl 2-(styrylcarbamoyl)malonates S2

Synthesis and spectral data of (E)-4-hydroxy-2-oxo-1,2-dihydropyridine-3- carboxamides S3

Synthesis and spectral data of Ethyl 4-hydroxy-2-oxo-1,2-dihydropyridine-3-carboxylates S4

Synthesis and spectral data of (E)-ethyl 2-((E)-styrylcarbamoyl)- 3-hydroxybut-2-enoates S6

$\begin{array}{ll}\text { Synthesis and spectral data of 3-acetyl-4-hydroxypyridin-2(1H)-ones } & \text { S7 }\end{array}$

${ }^{1} \mathrm{H}$ NMR and ${ }^{13} \mathrm{C}$ NMR spectra of (E)-diethyl 2-(styrylcarbamoyl)malonates $\quad$ S7

${ }^{1} \mathrm{H}$ NMR and ${ }^{13} \mathrm{C}$ NMR spectra of (E)-4-hydroxy-2-oxo-1,2-dihydropyridine-3-carboxamides S11

${ }^{1} \mathrm{H}$ NMR and ${ }^{13} \mathrm{C}$ NMR spectra of Ethyl 4-hydroxy-2-oxo-1,2-dihydropyridine-3-carboxylates S15

${ }^{1} \mathrm{H}$ NMR and ${ }^{13} \mathrm{C}$ NMR spectra of (E)-ethyl 2-((E)-styrylcarbamoyl)-3-hydroxybut-2-enoates S18

${ }^{1} \mathrm{H}$ NMR and ${ }^{13} \mathrm{C}$ NMR spectra of 3-acetyl-4-hydroxypyridin-2(1H)-ones $\quad$ S20

X-ray crystallographic data for compound ethyl 2-(((E)-4-methoxystyryl)carbamoyl)

$\begin{array}{lr}-3 \text {-hydroxybut-2-enoate } & \text { S22 }\end{array}$ 


\section{General information}

Melting points were determined with RY-1 apparatus, and were uncorrected. IR spectra were determined as $\mathrm{KBr}$ pellets on a Shimadzu model 470 spectrophotometer. ${ }^{1} \mathrm{H}$ NMR spectra were recorded using a Bruker $\mathrm{AV} 400 \mathrm{MHz}$ spectrometer in DMSO- $d_{6}$ and $\mathrm{CDCl}_{3}$ with tetramethylsilane as internal standard. EI mass spectra were recorded on Shimadzu QP-2010 GC-MS system and Waters Micromass GCT system. ESI mass spectra were obtained on Shimadzu 2010A LC-MS instrument. Crystal structure was determined by using a Bruker SMART CCD area detector diffractometer. All chemicals and reagents were purchased from known commercial suppliers and were used without further purification. (E)-diethyl 2-(styrylcarbamoyl)malonate were synthesized according to the literature procedures with minor change ${ }^{[10]}$.

\section{Synthesis and spectral data of (E)-diethyl 2-(styrylcarbamoyl)malonates}

General procedure for the preparation of (E)-diethyl 2-(styrylcarbamoyl)malonate 5. To a ice-cooled solution of cinnamic acid $(10.0 \mathrm{~g}, 67.6 \mathrm{mmol})$ in $100 \mathrm{~mL}$ toluene was added triethylamine (10.3 mL, $74.7 \mathrm{mmol})$ and diphenyl phosphorazidate (DPPA, $18.6 \mathrm{~g}, 67.6 \mathrm{mmol})$. The reactant solution was stirred at room temperature for $3 \mathrm{~h}$. The acyl azide product was isolated by dilution with cold water. The organic layer was dried over anhydrous magnesium sulfate, and the solvent was removed in vacuo to provide crude product. The acyl azide was dissolved in 20 $\mathrm{mL}$ of benene and heated at reflux until azide decomposition was complete as monitored by TLC. The reaction mixture was then cooled to $0^{\circ} \mathrm{C}$ and diethylsodiomalonate (prepared from diethyl malonate $(10.3 \mathrm{~g}, 67.6 \mathrm{mmol})$ and sodium hydride $(2.23 \mathrm{~g}, 80 \%$ dispersion in oil, $74.3 \mathrm{mmol})$ in toluene $(100 \mathrm{~mL})$ at $\left.0^{\circ} \mathrm{C}\right)$ was added. The reaction mixture was allowed to warm to room temperature over $2 \mathrm{~h}$, quenched with a saturated aqueous ammonium chloride solution, extracted with ether $(3 \times 100 \mathrm{~mL})$, rinsed with brine $(3 \times 100 \mathrm{~mL})$, and dried over with anhydrous sodium sulfate. The solvent was removed in vacuo to give the compound $\mathbf{5}$ as white crystals. The physical and spectral data of the compounds $\mathbf{5 a - 5 d}$ are as follows.

(E)-diethyl 2-(styrylcarbamoyl)malonate (5a). Yield 83\%. White solid, M.p 78-80 ${ }^{\circ} \mathrm{C} . \mathrm{IR}(\mathrm{KBr}$, $\left.v \mathrm{~cm}^{-1}\right): 3267,1745,1652,1310,1176,1036,924,693 .{ }^{1} \mathrm{H} \mathrm{NMR}\left(400 \mathrm{MHz}, \mathrm{CDCl}_{3}\right) \delta=1.30(\mathrm{t}, J=$ $7.1 \mathrm{~Hz}, 3 \mathrm{H}), 1.32$ (t, $J=7.1 \mathrm{~Hz}, 3 \mathrm{H}), 4.29$ (t, $J=10.7 \mathrm{~Hz}, 4 \mathrm{H}), 4.41$ (s, 1H), 6.24 (d, $J=14.7 \mathrm{~Hz}$, 1H), 7.18-7.21 (m, 1H), 7.30-7.45(m, 4H), 7.49-7.52 (m, 1H),9.22 (d, J=10.3 Hz, 1H). ${ }^{13} \mathrm{C}$ NMR (101 MHz, $\left.\mathrm{CDCl}_{3}\right): \delta=13.9,58.7,63.0,115.1,121.7,125.7,126.9,128.6,135.7,159.4,165.4$. 
HRMS-EI: [M] ${ }^{+}$Calc. for $\mathrm{C}_{16} \mathrm{H}_{19} \mathrm{NO}_{5} 305.1263$, Found 305.1262.

(E)-diethyl 2-((4-methoxystyryl)carbamoyl)malonate (5b). Yield 78\%. White solid, M.p $83-85^{\circ} \mathrm{C} . \mathrm{IR}\left(\mathrm{KBr}, v \mathrm{~cm}^{-1}\right): 3307,1743,1660,1507,1309,1253,1179,1033,945,851,814 .{ }^{1} \mathrm{H}$ NMR (400 MHz, $\left.\mathrm{CDCl}_{3}\right): \delta=1.32(\mathrm{t}, \mathrm{J}=7.1 \mathrm{~Hz}, 6 \mathrm{H}), 3.80(\mathrm{~s}, 3 \mathrm{H}), 4.27-4.32(\mathrm{~m}, 4 \mathrm{H}), 4.40(\mathrm{~s}$, 1H), $6.24(\mathrm{~d}, \mathrm{~J}=14.7 \mathrm{~Hz}, 1 \mathrm{H}), 6.86(\mathrm{~d}, \mathrm{~J}=8.8 \mathrm{~Hz}, 2 \mathrm{H}), 7.25-7.28(\mathrm{~m}, 2 \mathrm{H}), 7.35(\mathrm{dd}, \mathrm{J}=14.7$, $10.6 \mathrm{~Hz}, 1 \mathrm{H}), 9.16(\mathrm{~d}, \mathrm{~J}=10.1 \mathrm{~Hz}, 1 \mathrm{H})$. HRMS-EI: $[\mathrm{M}]^{+}$Calc. for $\mathrm{C}_{17} \mathrm{H}_{21} \mathrm{NO}_{6} 335.1369$, Found 335.1362. This compound was reported in literature [10a].

(E)-diethyl 2-(4-chlorostyrylcarbamoyl)malonate (5c). Yield 87\%. White solid, M.p. $81-83^{\circ} \mathrm{C} \operatorname{IR}\left(\mathrm{KBr}, v \mathrm{~cm}^{-1}\right): 3269,3079,2983,1760,1748,1731,1648,1548,1490,1307,1263$, 1155, 1033, 966, 856,814, 638. ${ }^{1} \mathrm{H}$ NMR $\left(400 \mathrm{MHz}, \mathrm{CDCl}_{3}\right): \delta=1.32(\mathrm{t}, \mathrm{J}=7.1 \mathrm{~Hz}, 6 \mathrm{H})$, 4.27-4.33 (m, 4H), $4.41(\mathrm{~s}, 1 \mathrm{H}), 6.20(\mathrm{~d}, \mathrm{~J}=14.6 \mathrm{~Hz}, 1 \mathrm{H}), 7.26-7.29(\mathrm{~m}, 4 \mathrm{H}), 7.46(\mathrm{dd}, \mathrm{J}=14.6$, $10.7 \mathrm{~Hz}, 1 \mathrm{H}), 9.27(\mathrm{~d}, \mathrm{~J}=10.5 \mathrm{~Hz}, 1 \mathrm{H}) .{ }^{13} \mathrm{C} \mathrm{NMR}\left(101 \mathrm{MHz}, \mathrm{CDCl}_{3}\right): \delta=13.9,58.6,63.0,113.9$, 122.2, 126.9, 128.8, 132.5, 134.3, 159.5, 165.4. HRMS-EI: Calc. for $\mathrm{C}_{16} \mathrm{H}_{18} \mathrm{ClNO}_{5}[\mathrm{M}]^{+}$ 339.0874, Found 339.0871.

(E)-diethyl 2-((4-nitrostyryl)carbamoyl)malonate (5d). Yield 81\%. White solid, M.p. $86-88^{\circ} \mathrm{C} . \mathrm{IR}\left(\mathrm{KBr}, v \mathrm{~cm}^{-1}\right): 3311,2986,1642,1603,1521,1382,1343,1171,1099,1007,948,860$, 803, 746. ${ }^{1} \mathrm{H}$ NMR $\left(400 \mathrm{MHz}, \mathrm{CDCl}_{3}\right): \delta=1.33(\mathrm{t}, \mathrm{J}=7.1 \mathrm{~Hz}, 6 \mathrm{H}), 4.28-4.30(\mathrm{~m}, 4 \mathrm{H}), 4.44(\mathrm{~s}$, 1H), $6.31(\mathrm{~d}, \mathrm{~J}=14.7 \mathrm{~Hz}, 1 \mathrm{H}), 7.44(\mathrm{~d}, \mathrm{~J}=8.8 \mathrm{~Hz}, 2 \mathrm{H}), 7.64(\mathrm{dd}, \mathrm{J}=14.6,10.8 \mathrm{~Hz}, 1 \mathrm{H}), 8.16(\mathrm{~d}$, $\mathrm{J}=8.8 \mathrm{~Hz}, 2 \mathrm{H}), 9.45(\mathrm{~d}, \mathrm{~J}=10.5 \mathrm{~Hz}, 1 \mathrm{H}) .{ }^{13} \mathrm{C} \mathrm{NMR}\left(101 \mathrm{MHz}, \mathrm{CDCl}_{3}\right): \delta=13.9,58.6,63.1$, 112.7, 124.2, 125.5, 125.9, 142.8, 146.3, 159.9, 165.2. HRMS-EI: $[M]^{+}$Calc. for $\mathrm{C}_{18} \mathrm{H}_{16} \mathrm{~N}_{2} \mathrm{O}_{7}$ 350.1114 , Found 350.1118 .

\section{Synthesis and spectral data of (E)-4-hydroxy-2-oxo-1,2-dihydropyridine-3-carboxamides}

General Procedure for the Preparation of (E)-4-hydroxy-2-oxo-1,2-dihydropyridine-3carboxamide 6. Compounds $5(1.3 \mathrm{mmol})$ were stirred in diphenyl ether $(5 \mathrm{~mL})$ at $200-210^{\circ} \mathrm{C}$ for $5 \mathrm{~min}$. The reaction mixture was cooled to room temperature. The precipitate was collected and washed with ethyl acetate and n-hexane (1:2) to give the compound $\mathbf{6}$ as white solid. It was performed for further spectrum analysis.

(E)-4-hydroxy-2-oxo-5-phenyl-N-styryl-1,2-dihydropyridine-3-carboxamide (6a). Yield 62\%. White solid, M.p. $258-260^{\circ} \mathrm{C}$, IR ( $\left.\mathrm{KBr}, v \mathrm{~cm}^{-1}\right): 3331,3030,2850,1665,1587,1287,752,692 .{ }^{1} \mathrm{H}$ NMR (400 MHz, DMSO-d $\left.{ }_{6}\right): \delta=6.50(\mathrm{~d}, J=10.8 \mathrm{~Hz}, 1 \mathrm{H}), 7.19(\mathrm{~s}, 1 \mathrm{H}), 7.52-7.21(\mathrm{~m}, 9 \mathrm{H}), 7.57(\mathrm{t}$, s3 
$J=10.8 \mathrm{~Hz}, 1 \mathrm{H}), 7.67(\mathrm{~s}, 1 \mathrm{H}), 12.27(\mathrm{~s}, 1 \mathrm{H}), 12.35(\mathrm{~d}, 1 \mathrm{H}), 15.90(\mathrm{~s}, 1 \mathrm{H}) .{ }^{13} \mathrm{C}$ NMR $(101 \mathrm{MHz}$, DMSO-d $\left.\mathrm{d}_{6}\right): \delta=97.7,114.2,116.0,121.3,126.1,127.3,127.8,128.7,129.1,129.7,133.2,136.3$, 139.2, 163.1, 168.5, 173.4. MS(EI, 70eV): $\mathrm{m} / \mathrm{z}=333(\mathrm{M}+1), 332\left(\mathrm{M}^{+}\right), 214,120,119,118,117,91$. HRMS(EI): Calc. for $\mathrm{C}_{20} \mathrm{H}_{16} \mathrm{~N}_{2} \mathrm{O}_{3}[\mathrm{M}]^{+} 332.1161$, Found 332.1162.

(E)-4-hydroxy-5-(4-methoxyphenyl)-N-(4-methoxystyryl)-2-oxo-1,2-dihydropyridine-3 -carboxamide (6b). Yield 70\%. White solid, M.p. $259-262^{\circ} \mathrm{C} . \operatorname{IR}\left(\mathrm{KBr}, v \mathrm{~cm}^{-1}\right): 2997,2839,1672$, 1642, 1610, 1552, 1509, 1448, 1283, 1247, 1177, 1030, 953, 834, $813 .{ }^{1} \mathrm{H}$ NMR (400 MHz, DMSO-d $\left._{6}\right): 3.75(\mathrm{~s}, 3 \mathrm{H}), 3.78(\mathrm{~s}, 3 \mathrm{H}), 6.46(\mathrm{~d}, \mathrm{~J}=14.7 \mathrm{~Hz}, 1 \mathrm{H}), 6.89(\mathrm{~d}, \mathrm{~J}=8.7 \mathrm{~Hz}, 2 \mathrm{H}), 6.97(\mathrm{~d}$, $\mathrm{J}=8.8 \mathrm{~Hz}, 2 \mathrm{H}), 7.36-7.44(\mathrm{~m}, 4 \mathrm{H}), 7.44-7.50(\mathrm{dd}, 1 \mathrm{H}), 7.62(\mathrm{~d}, \mathrm{~J}=6.3 \mathrm{~Hz}, 1 \mathrm{H}), 12.21(\mathrm{~d}, \mathrm{~J}=$ $6.2 \mathrm{~Hz}, 1 \mathrm{H}), 12.35$ (d, J = 10.5 Hz, 1H), 15.99 (s, 1H). ${ }^{13} \mathrm{C}$ NMR (101 MHz, DMSO-d 6 ) : 55.5, 55.6, 97.7, 114.0, 114.1, 114.6, 115.9, 119.5, 125.4, 127.3, 128.7, 130.6, 138.4, 158.8, 159.1, 163.0, 168.2, 173.4. HRMS(EI): Calc. for $\mathrm{C}_{22} \mathrm{H}_{20} \mathrm{~N}_{2} \mathrm{O}_{5}[\mathrm{M}]^{+}$392.1372, Found392.1377.

(E)-4-hydroxy-5-(4-chlorophenyl)-N-(4-chlorostyryl)-2-oxo-1,2-dihydropyridine-3-carboxamide (6c).Yield 55\%. White solid, M.p. 280-281 ${ }^{\circ} \mathrm{C}, \operatorname{IR}\left(\mathrm{KBr}, v \mathrm{~cm}^{-1}\right): 3129,2987,2853,1671$, 1539, 1483, 1457, 1398, 1242, 1092, 952, 861, 827, $756 .{ }^{1} \mathrm{H}$ NMR (400 MHz, DMSO-d 6 ): $\delta=6.53(\mathrm{~d}, \mathrm{~J}=14.7 \mathrm{~Hz}, 1 \mathrm{H}), 7.36(\mathrm{~d}, \mathrm{~J}=8.4 \mathrm{~Hz}, 2 \mathrm{H}), 7.48(\mathrm{dd}, \mathrm{J}=8.1,3.7 \mathrm{~Hz}, 4 \mathrm{H}), 7.54(\mathrm{~d}, \mathrm{~J}=$ $8.5 \mathrm{~Hz}, 2 \mathrm{H}), 7.62(\mathrm{dd}, \mathrm{J}=14.6,10.7 \mathrm{~Hz}, 1 \mathrm{H}), 7.76(\mathrm{~d}, \mathrm{~J}=5.9 \mathrm{~Hz}, 1 \mathrm{H}), 12.36(\mathrm{~s}, 1 \mathrm{H}), 12.38$ (s, 1H), $15.95(\mathrm{~s}, 1 \mathrm{H}), .{ }^{13} \mathrm{C}$ NMR $\left(101 \mathrm{MHz}, \mathrm{DMSO}-\mathrm{d}_{6}\right): \delta=97.7,112.9,114.9,122.1,127.8,128.6$, $129.1,131.2,131.5,132.0,132.6,135.4,139.5,163.1,168.5,173.2$. HRMS(EI): Calc. for $\mathrm{C}_{20} \mathrm{H}_{14} \mathrm{Cl}_{2} \mathrm{~N}_{2} \mathrm{O}_{3}[\mathrm{M}]^{+}$400.0381, Found 400.0378.

(E)-4-hydroxy-5-(4-nitrophenyl)-N-(4-nitrostyryl)-2-oxo-1,2-dihydropyridine-3-carboxamide (6d). Yield 51\%. White solid, M.p.294-296 ${ }^{\circ} \mathrm{C}$, IR $\left(\mathrm{KBr}, v_{\mathrm{cm}}^{-1}\right): 3333,1682,1638,1542,1506$, 1453, 1325, 1103, 957, 853, 748, 690. ${ }^{1} \mathrm{H}$ NMR (400 MHz, DMSO-d 6 ): $\delta=6.73$ (d, J = $14.6 \mathrm{~Hz}$, 1H), $7.74(\mathrm{~d}, \mathrm{~J}=8.7 \mathrm{~Hz}, 2 \mathrm{H}), 7.85(\mathrm{~d}, \mathrm{~J}=8.7 \mathrm{~Hz}, 2 \mathrm{H}), 7.87-7.93(\mathrm{~m}, 1 \mathrm{H}), 7.98(\mathrm{~d}, \mathrm{~J}=6.7 \mathrm{~Hz}$, $1 \mathrm{H}), 8.17(\mathrm{~d}, \mathrm{~J}=8.8 \mathrm{~Hz}, 2 \mathrm{H}), 8.28(\mathrm{~d}, \mathrm{~J}=8.7 \mathrm{~Hz}, 2 \mathrm{H}), 12.46(\mathrm{~d}, \mathrm{~J}=10.6 \mathrm{~Hz}, 1 \mathrm{H}), 12.61(\mathrm{~d}, \mathrm{~J}=$ $6.8 \mathrm{~Hz}, 1 \mathrm{H}), 15.96(\mathrm{~s}, 1 \mathrm{H}) .{ }^{13} \mathrm{C}$ NMR $\left(101 \mathrm{MHz}, \mathrm{DMSO}-\mathrm{d}_{6}\right): \delta=97.2,111.4,113.6,123.2,123.9$, 125.0, 126.3, 129.8, 139.6, 140.5, 143.3, 145.4, 146.2, 162.6, 168.3, 172.6. HRMS(EI): Calc. for: $\mathrm{C}_{20} \mathrm{H}_{14} \mathrm{~N}_{4} \mathrm{O}_{7}[\mathrm{M}]^{+}$422.0862, Found 422.0867 .

\section{Synthesis and spectral data of Ethyl 4-hydroxy-2-oxo-1,2-dihydropyridine-3-carboxylates}

General procedure for the preparation of ethyl 
4-hydroxy-2-oxo-1,2-dihydropyridine-3-carboxylate 7 . Compounds 5 (1.3 mmol) were refluxed in xylene $(20 \mathrm{~mL})$ for 12 hours. The solvent was removed in vacuo to give an oil. The residue was purified by column chromatography (ethyl acetate:hexane=1:6) to give the compound 7 a white solid.

Ethyl 4-hydroxy-5-phenyl-2-oxo-1,2-dihydropyridine-3-carboxylate (7a).Yield 60\%. White solid, m.p. 187 190 ${ }^{\circ} \mathrm{C}$, colorless solid, IR $\left(\mathrm{KBr}, v \mathrm{~cm}^{-1}\right): 3125: 2981,2358,1660,1548,1200$, 813, 763, 697. ${ }^{1} \mathrm{H}$ NMR (400 MHz, DMSO-d $\left.{ }_{6}\right): \delta=1.30(\mathrm{t}, 3 \mathrm{H}), 4.53(\mathrm{q}, 2 \mathrm{H}), 7.32-7.34(\mathrm{~m}, 1 \mathrm{H})$, 7.38-7.41(m, 2H), 7.44-7.46(m, 2H), 7.59(s, 1H), 11.71(s, 1H), 13.67(s, 1H). ${ }^{13} \mathrm{C}$ NMR $(101$ MHz, DMSO-d $\left.{ }_{6}\right): \delta=14.5,61.7,98.8,111.9,127.6,128.6,129.4,133.6,140.0,159.7,172.3$, 172.6. $\mathrm{MS}(\mathrm{EI}, 70 \mathrm{eV}): \mathrm{m} / \mathrm{z}=259\left(\mathrm{M}^{+}\right), 214,213,145,144,119,112,89$. HRMS: Calc. for $\mathrm{C}_{14} \mathrm{H}_{13} \mathrm{NO}_{4}[\mathrm{M}]^{+}$259.0845, Found.259.0847. This compound was reported in literature [16].

Ethyl 4-hydroxy-5-(4-methoxyphenyl)-2-oxo-1,2-dihydropyridine-3-carboxylate (7b).Yield 71\%. White solid, M.p. $\quad 191-193 \quad{ }^{\circ} \mathrm{C} \quad, \quad \operatorname{IR}\left(\mathrm{KBr}, v \mathrm{~cm}^{-1}\right)$ : 3446 , 2962,2842,1673,1638,1246,1077,1507,1441,833,807. ${ }^{1} \mathrm{H}$ NMR (400 MHz, DMSO-d $\left.{ }_{6}\right): \delta=1.30(\mathrm{t}$, $J=6.9 \mathrm{~Hz}, 3 \mathrm{H}), 3.77(\mathrm{~s}, 3 \mathrm{H}), 4.34(\mathrm{q}, J=6.9 \mathrm{~Hz}, 2 \mathrm{H}), 6.96(\mathrm{~d}, J=7.9 \mathrm{~Hz}, 2 \mathrm{H}), 7.36(\mathrm{~d}, J=7.6$ $\mathrm{Hz}, 2 \mathrm{H}), 7.59$ (s, 1H), 11.66 (s, 1H), 13.66 (s, 1H). ${ }^{13} \mathrm{C}$ NMR (101 MHz, DMSO-d $\left.\mathrm{d}_{6}\right): \delta=14.5$, 55.5, 61.6, 98.7, 111.7, 114.1, 125.8, 130.6, 140.2, 158.9, 159.6, 172.4, 172.8. HRMS: Calc. for $\mathrm{C}_{15} \mathrm{H}_{15} \mathrm{NO}_{5}[\mathrm{M}]^{+}$289.0950, Found 289.0952. This compound was reported in literature [12a].

Ethyl 4-hydroxy-5-(4-chlorophenyl)-2-oxo-1,2-dihydropyridine-3-carboxylate (7c). Yield 54\%. White solid, M.p. 233-235 ${ }^{\circ} \mathrm{C}, \operatorname{IR}\left(\mathrm{KBr}, v_{\mathrm{cm}}{ }^{-1}\right): 2977,2855,1667,1542,1491,1439,1396$, 1244, 1198, 1092, 826. ${ }^{1} \mathrm{H}$ NMR $\left(400 \mathrm{MHz}, \mathrm{DMSO}_{6}\right): \delta=1.31(\mathrm{t}, \mathrm{J}=7.1 \mathrm{~Hz}, 3 \mathrm{H}), 4.34(\mathrm{q}, \mathrm{J}=$ 7.1 Hz, 2H), 7.44-7.46 (m, 2H), 7.48-7.50 (m, 2H), $7.64(\mathrm{~s}, 1 \mathrm{H}), 11.75(\mathrm{~s}, 1 \mathrm{H}), 13.68(\mathrm{~s}, 1 \mathrm{H}) .{ }^{13} \mathrm{C}$ NMR (101 MHz, DMSO-d 6 ): $\delta=14.5,61.7,98.8,110.71,128.6,131.2,132.3,132.5,141.0,159.7$, 172.2, 172.4, MS(EI, 70eV): m/z=293, 247, 178, 136, 106, 91, HRMS: Calcd. for $\mathrm{C}_{14} \mathrm{H}_{12} \mathrm{ClN}_{2} \mathrm{O}_{4}$ $[\mathrm{M}]^{+}$293.0455, Found: 293.0460.

Ethyl 4-hydroxy-5-(4-nitrophenyl)-2-oxo-1,2-dihydropyridine-3-carboxylate (7d). Yield 48\%. White solid, M.p. $233-235^{\circ} \mathrm{C}$, IR( $\left(\mathrm{KBr}, v \mathrm{~cm}^{-1}\right): 3160,3045,2901,1672,1605,1509,1445,1248$, 1015, 853. ${ }^{1} \mathrm{H}$ NMR $\left(400 \mathrm{MHz}, \mathrm{DMSO}-\mathrm{d}_{6}\right): \delta=1.31(\mathrm{t}, J=7.1 \mathrm{~Hz}, 3 \mathrm{H}), 4.35(\mathrm{q}, J=7.1 \mathrm{~Hz}, 2 \mathrm{H})$, $7.78(\mathrm{~d}, J=8.6 \mathrm{~Hz}, 2 \mathrm{H}), 8.16(\mathrm{~d}, J=8.6 \mathrm{~Hz}, 1 \mathrm{H}), 8.25(\mathrm{~d}, J=8.7 \mathrm{~Hz}, 2 \mathrm{H}), 11.99(\mathrm{~s}, 1 \mathrm{H}), 13.83(\mathrm{~s}$, 1H). MS(EI, 70eV): m/z=304, 258, 232, 189, 156, 133,117,91, HRMS: Calcd. for $\mathrm{C}_{14} \mathrm{H}_{13} \mathrm{~N}_{2} \mathrm{O}_{6}$ : s5 
304.0695, Found: 304.0699 .

\section{Synthesis and spectral data of (E)-ethyl 2-((E)-styrylcarbamoyl)- 3-hydroxybut-2-enoates}

General procedure for the preparation of (E)-ethyl 2-((E)-styrylcarbamoyl)3-hydroxybut-2-enoate 12. To a ice-cooled solution of cinnamic acid $(2.0 \mathrm{~g}, 11.2 \mathrm{mmol})$ in 20 $\mathrm{mL}$ toluene was added triethylamine $(1.6 \mathrm{~mL}, 11.6 \mathrm{mmol})$ and diphenyl phosphorazidate (DPPA, $2.9 \mathrm{~g}, 10.5 \mathrm{mmol})$. The reactant solution was stirred at room temperature for $3 \mathrm{~h}$. The acyl azide product was isolated by dilution with cold water. The organic layer was dried over anhydrous magnesium sulfate, and the solvent was removed in vacuo to provide crude product. The acyl azide was dissolved in $20 \mathrm{~mL}$ of benene and heated at reflux until azide decomposition was complete as monitored by TLC. The reaction mixture was then cooled to $0{ }^{\circ} \mathrm{C}$ and ethylsodioacetocaetate (prepared from ethyl acetoacetic acid ethyl ester (1.46 g, $11.2 \mathrm{mmol})$ and sodium hydride $(0.37 \mathrm{~g}, 80 \%$ dispersion in oil , $12.3 \mathrm{mmol})$ in toluene $(10 \mathrm{~mL})$ at $\left.0{ }^{\circ} \mathrm{C}\right)$ was added. The reaction mixture was allowed to warm to room temperature over $2 \mathrm{~h}$, quenched with a saturated aqueous ammonium chloride solution extrated with ether $(3 \times 30 \mathrm{~mL})$, rinsed with brine $(3 \times 30 \mathrm{~mL})$, and dried over with anhydrous sodium sulfate. The solvent was removed in vacuo to give the compound $\mathbf{1 2}$ as white crystals.

(E)-ethyl 2-((E)-styrylcarbamoyl)-3-hydroxybut-2-enoate (12a).Yield $\quad 85 \%$. White solid,.M.p. $88-91^{\circ} \mathrm{C}$. IR $\left(\mathrm{KBr}, v \mathrm{~cm}^{-1}\right): 3233,2987,1738,1642,1530,958,693 .{ }^{1} \mathrm{H}$ NMR $(400$ $\left.\mathrm{MHz}, \mathrm{CDCl}_{3}\right): \delta=1.37(\mathrm{t}, 3 \mathrm{H}), 2.48(\mathrm{~s}, 3 \mathrm{H}), 4.29(\mathrm{q}, J=14.3 \mathrm{~Hz}, 2 \mathrm{H}), 6.22(\mathrm{~s}, 1 \mathrm{H}), 7.26-7.35(\mathrm{~m}$, 1H), 7.32-7.35 (m, $J=22.6 \mathrm{~Hz}, 4 \mathrm{H}), 7.51-7.53(\mathrm{~m}, 1 \mathrm{H}), 11.07(\mathrm{~s}, 1 \mathrm{H}), 18.10(\mathrm{~s}, 1 \mathrm{H}) . \mathrm{MS}(\mathrm{EI}$, $70 \mathrm{eV}): \mathrm{m} / \mathrm{z}=275\left(\mathrm{M}^{+}\right), 229,145,120,119,118,91,43, \mathrm{HRMS}(\mathrm{EI}):$ Calc. for $\mathrm{C}_{15} \mathrm{H}_{17} \mathrm{NO}_{4}[\mathrm{M}]^{+}$ 275.1158, Found 275.1154.

(E)-ethyl 2-(((E)-4-methoxystyryl)carbamoyl)-3-hydroxybut-2-enoate (12b). Yield 78\%. White solid, M.p. $91-93^{\circ} \mathrm{C}, \operatorname{IR}\left(\mathrm{KBr}, v \mathrm{~cm}^{-1}\right): 3161,3069,2986,1731,1639,1542,1410,961,841$, 789. ${ }^{1} \mathrm{H}$ NMR $\left(400 \mathrm{MHz}, \mathrm{CDCl}_{3}\right): \delta=1.39$ (t, $\left.J=7.1 \mathrm{~Hz}, 3 \mathrm{H}\right), 2.49$ (s, 3H), $3.82(\mathrm{~s}, 3 \mathrm{H}), 4.32$ (q, $J=7.1 \mathrm{~Hz}, 2 \mathrm{H}), 6.22(\mathrm{~d}, J=14.6 \mathrm{~Hz}, 1 \mathrm{H}), 6.87(\mathrm{~d}, J=8.8 \mathrm{~Hz}, 2 \mathrm{H}), 7.29(\mathrm{~d}, J=8.8 \mathrm{~Hz}, 2 \mathrm{H}), 7.43$ (dd, 1H), 11.05 (d, $J=10.5 \mathrm{~Hz}, 1 \mathrm{H}) . \mathrm{MS}(\mathrm{EI}, 70 \mathrm{eV}): \mathrm{m} / \mathrm{z}=305\left(\mathrm{M}^{+}\right), 259,175,150,149,134,132$, 43. This compound was reported in literature [10a]. Crystals of this compound suitable for X-ray diffraction were obtained by slow evaporation of ethyl acetate-petroleum ether solution (1:1).

(E)-ethyl 2-(((E)-4-chlorostyryl)carbamoyl)-3-hydroxybut-2-enoate (12c). Yield $\quad 72 \%$. 
White solid, M.p. $98-100^{\circ} \mathrm{C}, \operatorname{IR}\left(\mathrm{KBr}, v \mathrm{~cm}^{-1}\right): 3241,2981,1646,1516,1406,1302,1160,1106$, 1025, 949, 852, 791, ${ }^{1} \mathrm{H}$ NMR (400 MHz): $\delta=1.37$ (t, $\left.J=7.1 \mathrm{~Hz}, 3 \mathrm{H}\right), 2.48$ (s, 3H), 4.30 (q, $J=$ $7.1 \mathrm{~Hz}, 2 \mathrm{H}), 6.16(\mathrm{~d}, J=14.6 \mathrm{~Hz}, 1 \mathrm{H}), 7.26(\mathrm{dd}, 4 \mathrm{H}), 7.52$ (dd, $J=14.6,10.7 \mathrm{~Hz}, 1 \mathrm{H}), 11.11(\mathrm{~d}$, $J=10.5 \mathrm{~Hz}, 1 \mathrm{H}) .{ }^{13} \mathrm{C} \mathrm{NMR}\left(101 \mathrm{MHz}, \mathrm{CDCl}_{3}\right): \delta=14.2,26.2,60.9,94.7,114.0,121.9,126.8$, 128.8, 132.3, 134.6, 168.8, 169.7, 192.2. MS(EI, 70eV): $\mathrm{m} / \mathrm{z}=309,263,179,153,129,117,91$. HRMS(EI): Calc. for $\mathrm{C}_{15} \mathrm{H}_{16} \mathrm{ClNO}_{4}[\mathrm{M}]^{+}$309.0768, Found 309.0770.

\section{Synthesis and spectral data of 3-acetyl-4-hydroxypyridin-2(1H)-ones}

General procedure for the preparation of 3-acetyl-4-hydroxypyridin-2(1H)-one 13. Compounds $9(1.5 \mathrm{mmol})$ were stirred at diphenyl ether $(5 \mathrm{~mL})$ at $200-210^{\circ} \mathrm{C}$ for $10 \mathrm{~min}$. The reaction mixture was cooled to room temperature. The precipitate was collected to perform spectrum analysis.

3-acetyl-4-hydroxy-5-phenylpyridin-2(1H)-one (13a).Yield 69\%. White solid, M.p. 215-217 ${ }^{\circ} \mathrm{C} . \mathrm{IR}\left(\mathrm{KBr}, v \mathrm{~cm}^{-1}\right): 3331,3030,2850,1665,1587,1287,752,692 .{ }^{1} \mathrm{H}$ NMR (400 MHz, DMSO-d $\left.\mathrm{d}_{6}\right): \delta=2.67$ (s, 3H), $7.34(\mathrm{~s}, 1 \mathrm{H}), 7.39$ (d, $\left.J=10.8 \mathrm{~Hz}, 2 \mathrm{H}\right), 7.47$ (d, $\left.J=10.8 \mathrm{~Hz}, 2 \mathrm{H}\right)$, $7.70(\mathrm{~s}, 1 \mathrm{H}), 11.83(\mathrm{~s}, 1 \mathrm{H}), 16.57$ (s, 1H). MS(EI, 70eV): $\mathrm{m} / \mathrm{z}=229\left(\mathrm{M}^{+}\right), 214,170,141,118,106$, 91, 77. This compound was reported in literature [12a].

3-Acetyl-4-hydroxy-5-(4-methoxyphenyl)pyridin-2(1H)-one (13b). Yield 79\%. White solid, M.p.230 232 ${ }^{\circ} \mathrm{C}$, white solid, IR (KBr, $\left.v \mathrm{~cm}^{-1}\right)$ : 3035, 2926, 2358, 1662, 1598, 1483, 1240, 820, 799. ${ }^{1} \mathrm{H}$ NMR (400 MHz, $\left.\mathrm{CDCl}_{3}\right): \delta=2.79(\mathrm{~s}, 3 \mathrm{H}), 3.84(\mathrm{~s}, 3 \mathrm{H}), 6.96(\mathrm{~d}, J=8.8 \mathrm{~Hz}, 2 \mathrm{H})$, 7.36-7.41(m, 3H), $16.60(\mathrm{~s}, 1 \mathrm{H}) . \mathrm{MS}(\mathrm{EI}, 70 \mathrm{eV}): \mathrm{m} / \mathrm{z}=259\left(\mathrm{M}^{+}\right), 244,148,133,132,117$. This compound was reported in literature [10a].

3-Acetyl-4-hydroxy-5-(4-chlorophenyl)pyridin-2(1H)-one (13c).Yield 65\%. White solid, M.p. 269 270 ${ }^{\circ} \mathrm{C}$, white solid, IR(KBr, $\left.v \mathrm{~cm}^{-1}\right): 3450,2801,1660,1489,1448,1249,1179,1095,829$. ${ }^{1} \mathrm{H}$ NMR (400 MHz, DMSO-d $\left.{ }_{6}\right): \delta=2.66$ (s, 3H), 7.43-7.48 (m, 2H), 7.48-7.53 (m, 2H), 7.75 (d, $J$ $=6.2 \mathrm{~Hz}, 1 \mathrm{H}), 11.88(\mathrm{~s}, 1 \mathrm{H}), 16.64(\mathrm{~s}, 1 \mathrm{H}) .{ }^{13} \mathrm{C}$ NMR $\left(101 \mathrm{MHz}, \mathrm{DMSO}-\mathrm{d}_{6}\right): \delta=31.3,106.8$, 111.1, 128.6, 131.1, 132.2, 132.4, 142.5, 162.1, 175.3, 206.1. MS(EI, 70eV): $\mathrm{m} / \mathrm{z}=263,248,220$, 193, 178, 152, 136, 117, 101, 91. HRMS-EI(m/z): [M] Calcd. for $\mathrm{C}_{13} \mathrm{H}_{10} \mathrm{ClNO}_{3}: 263.0349$, Found: 263.0350.

\footnotetext{
${ }^{1} \mathrm{H}$ NMR and ${ }^{13} \mathrm{C}$ NMR spectra of (E)-diethyl 2-(styrylcarbamoyl)malonates 
(E)-diethyl 2-(styrylcarbamoyl)malonate (5a)
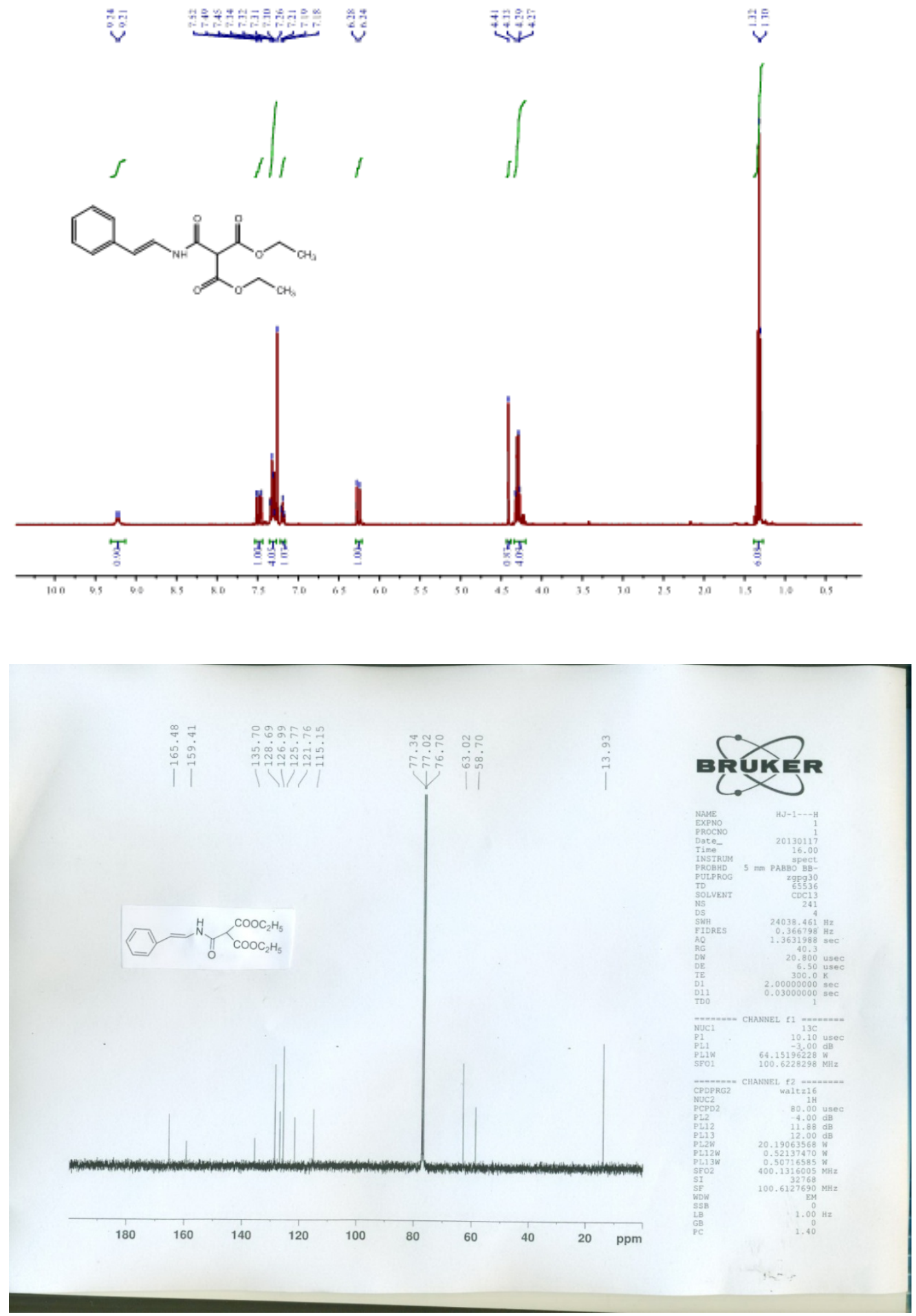

(E)-diethyl 2-(4-methoxystyrylcarbamoyl)malonate (5b) 


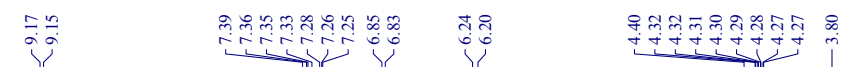

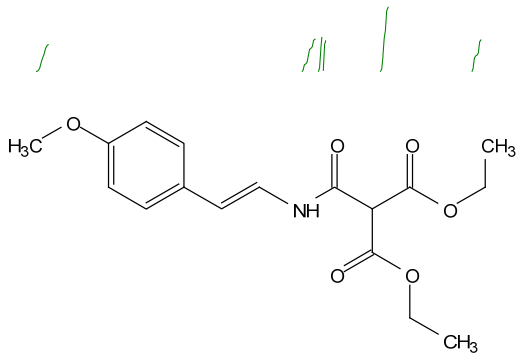

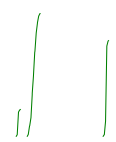
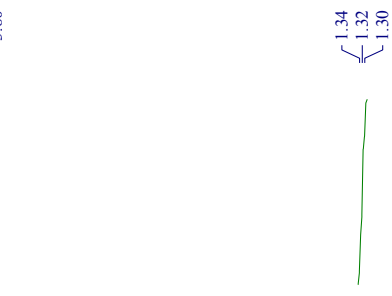

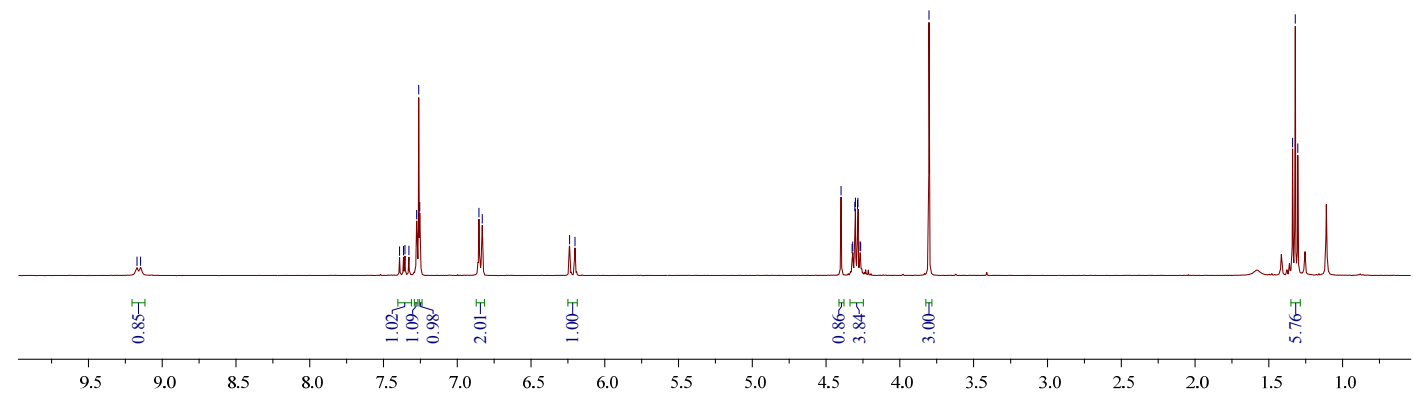

(E)-diethyl 2-(4-chlorostyrylcarbamoyl)malonate(5c)

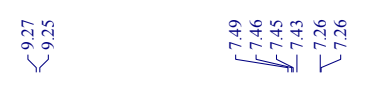

I

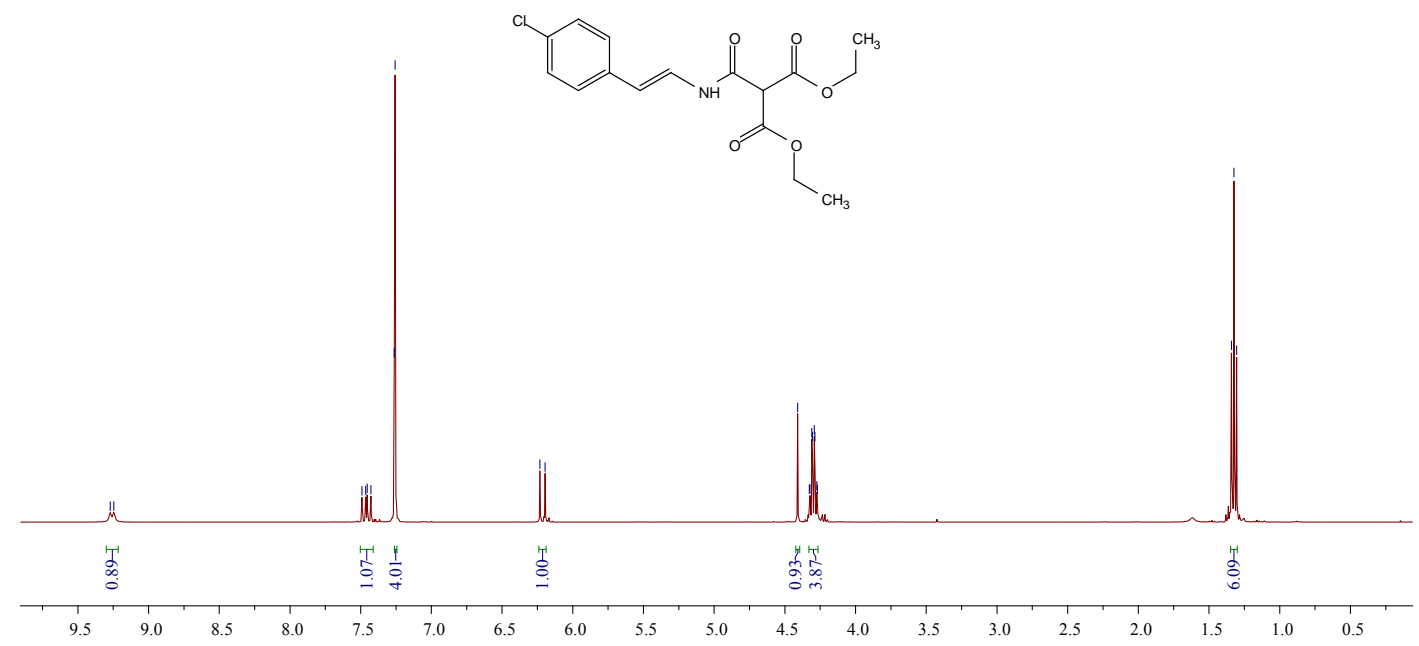

s9 


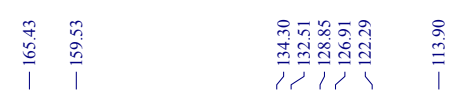

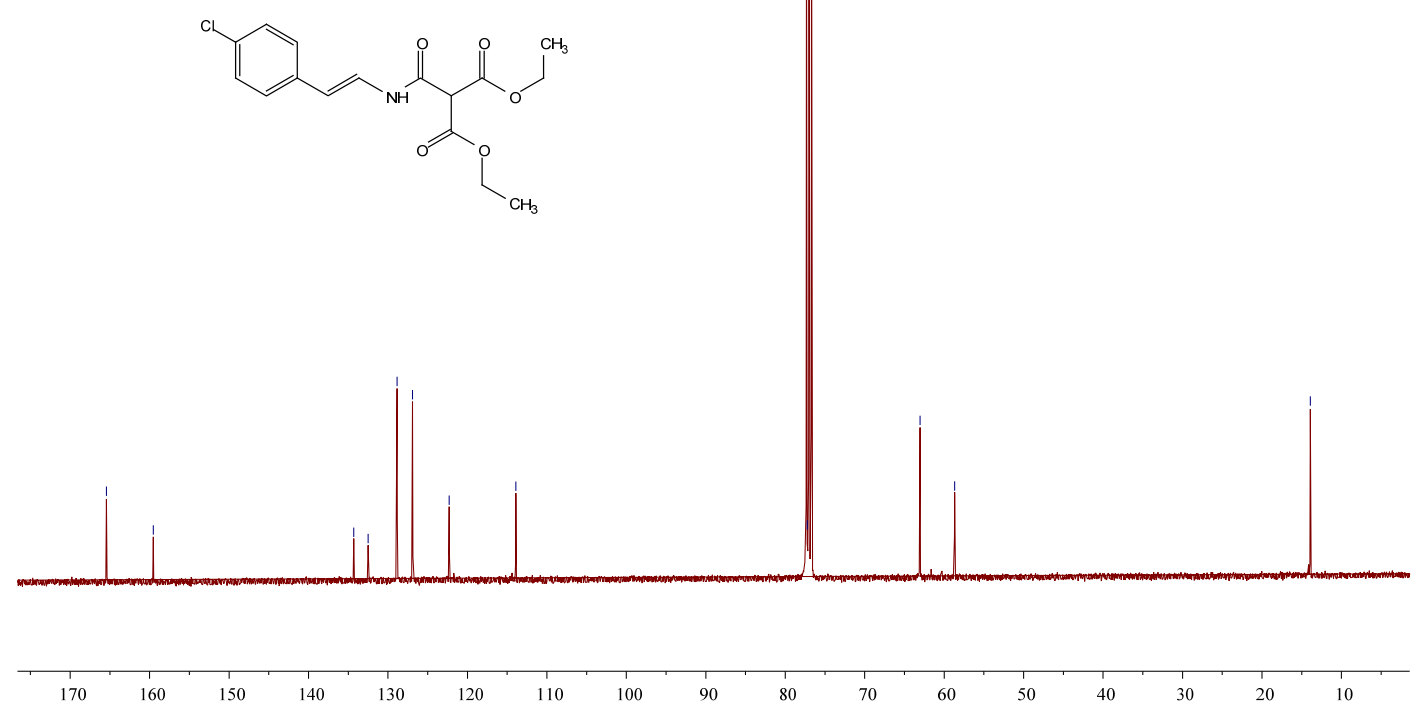

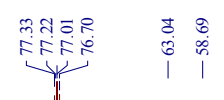

$\underset{\vec{a}}{\vec{j}}$

(E)-diethyl 2-(4-nitrostyrylcarbamoyl)malonate (5d)
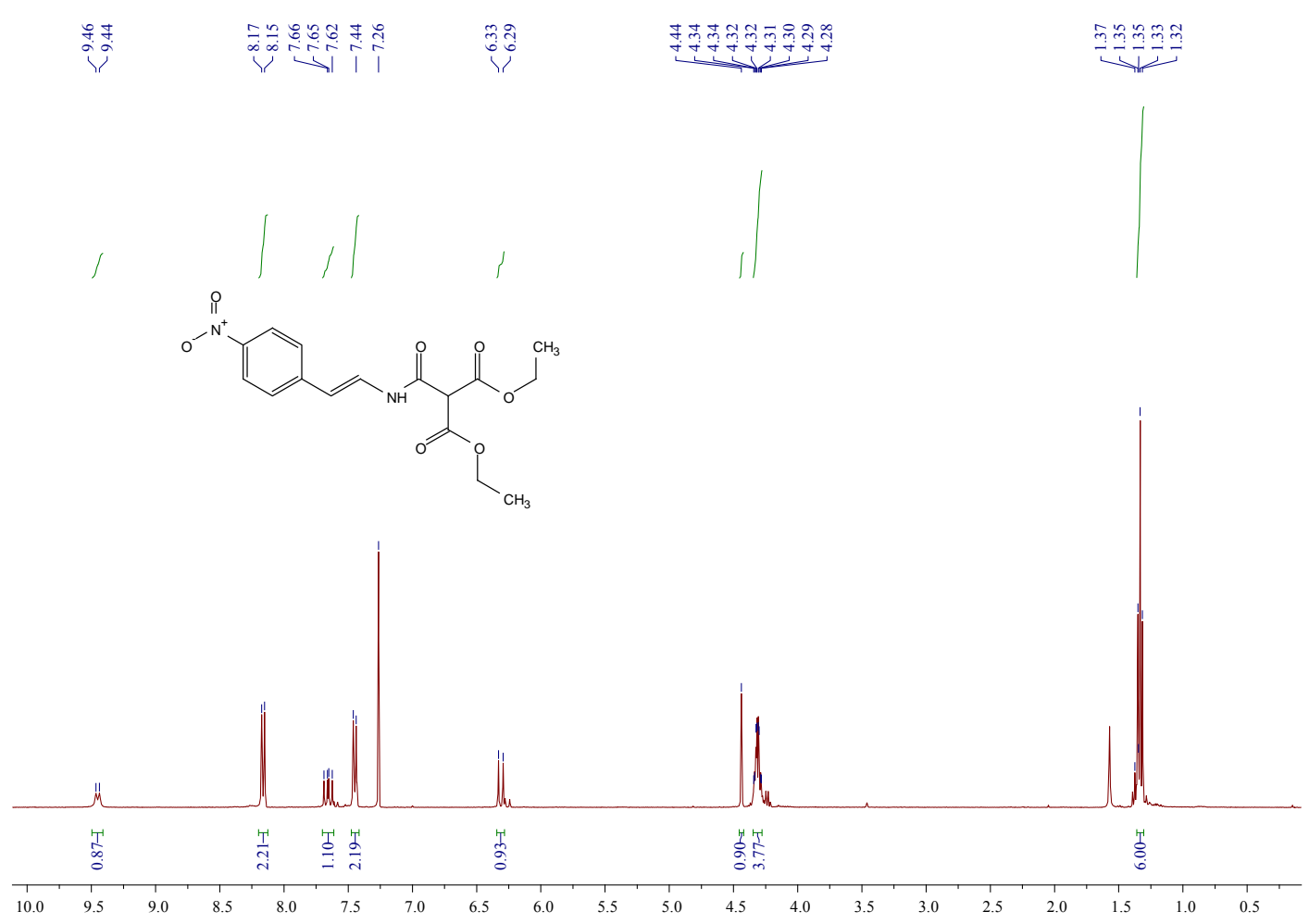

s10 


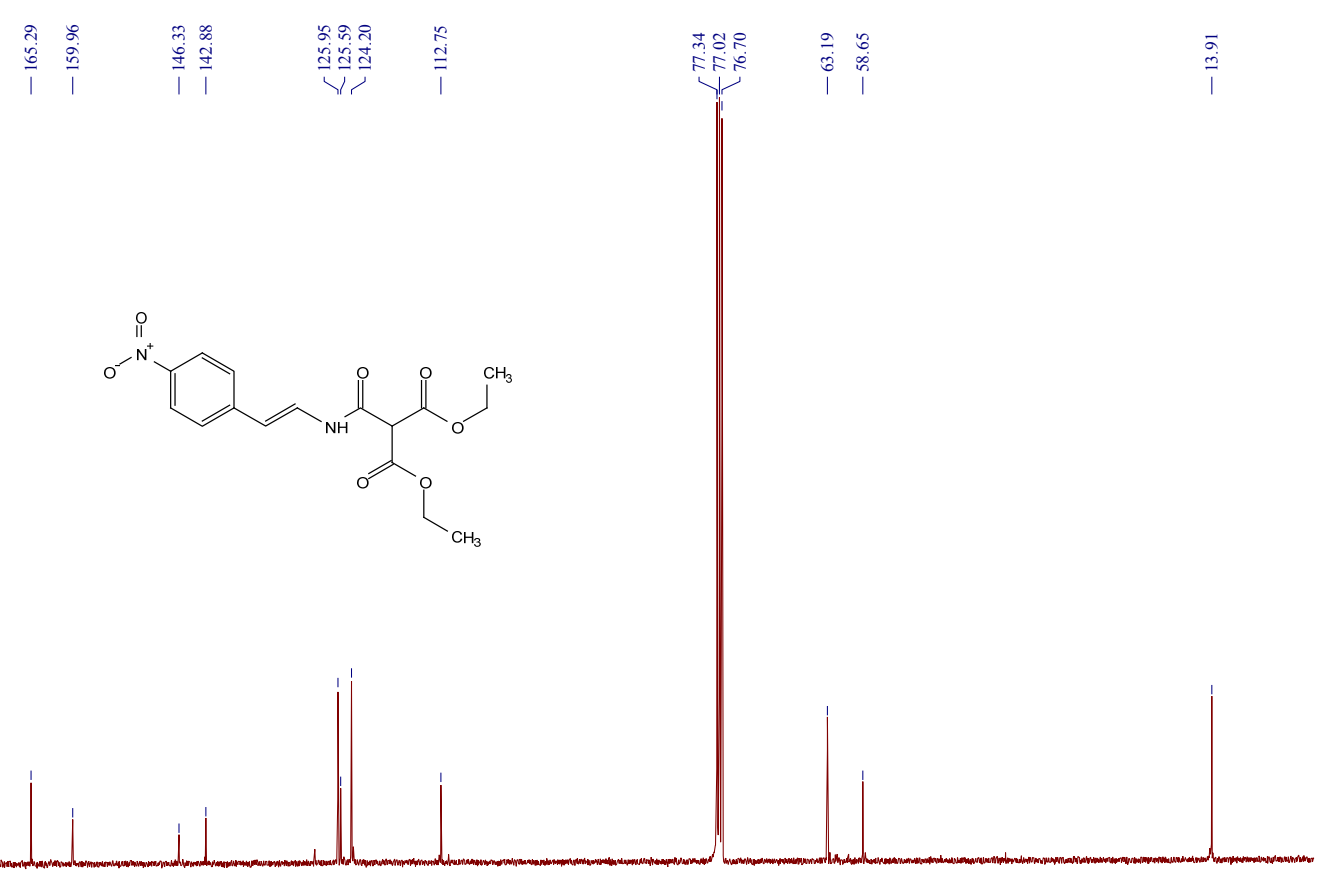

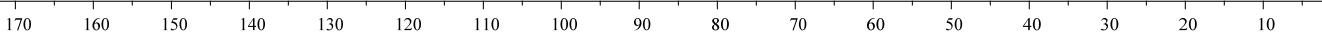

${ }^{1}$ H NMR and ${ }^{13} \mathrm{C}$ NMR spectra of (E)-4-hydroxy-2-oxo-1,2-dihydropyridine-3- carboxamides

(E)-4-hydroxy-2-oxo-5-phenyl-N-styryl-1,2-dihydropyridine-3-carboxamide (6a)
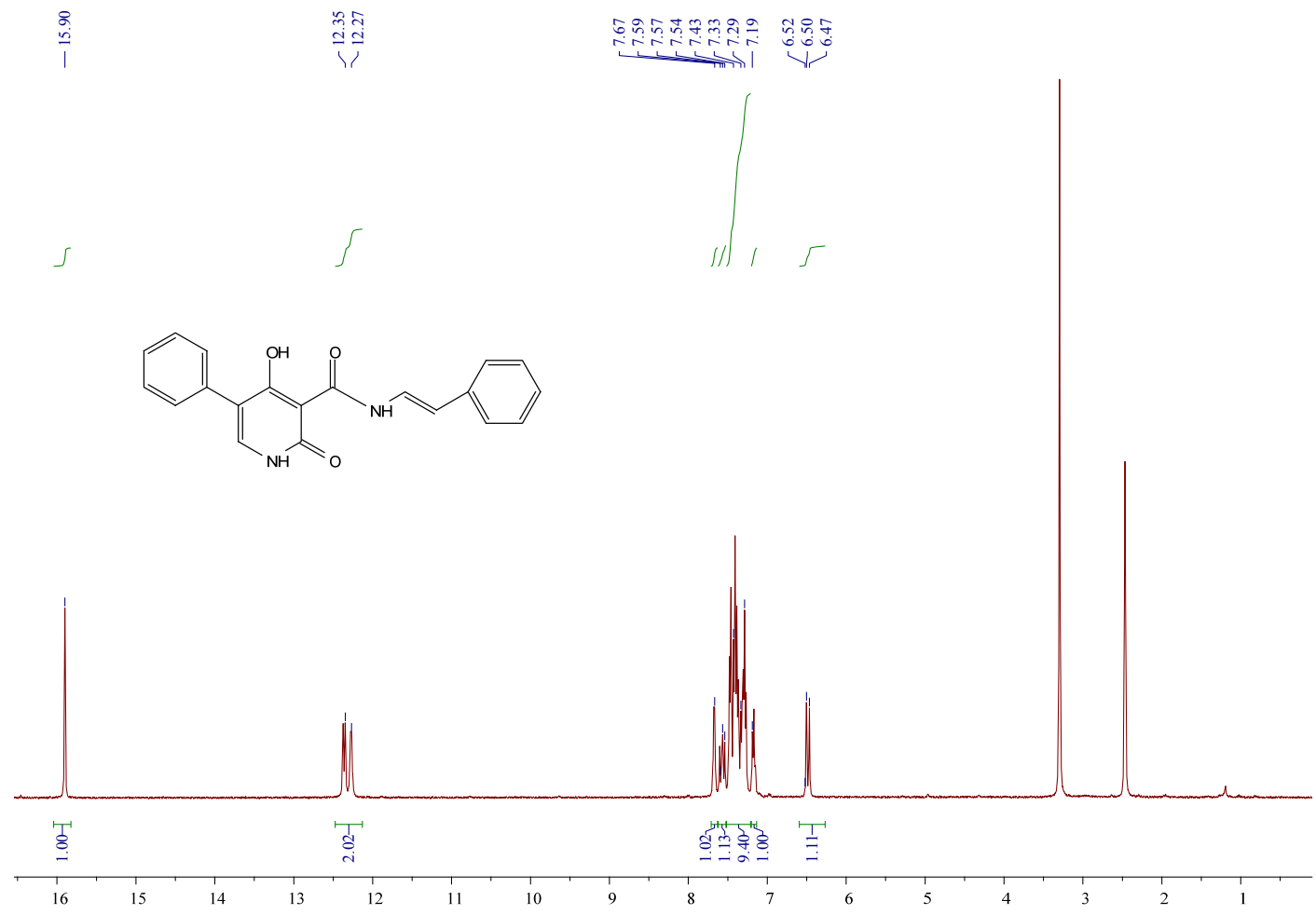

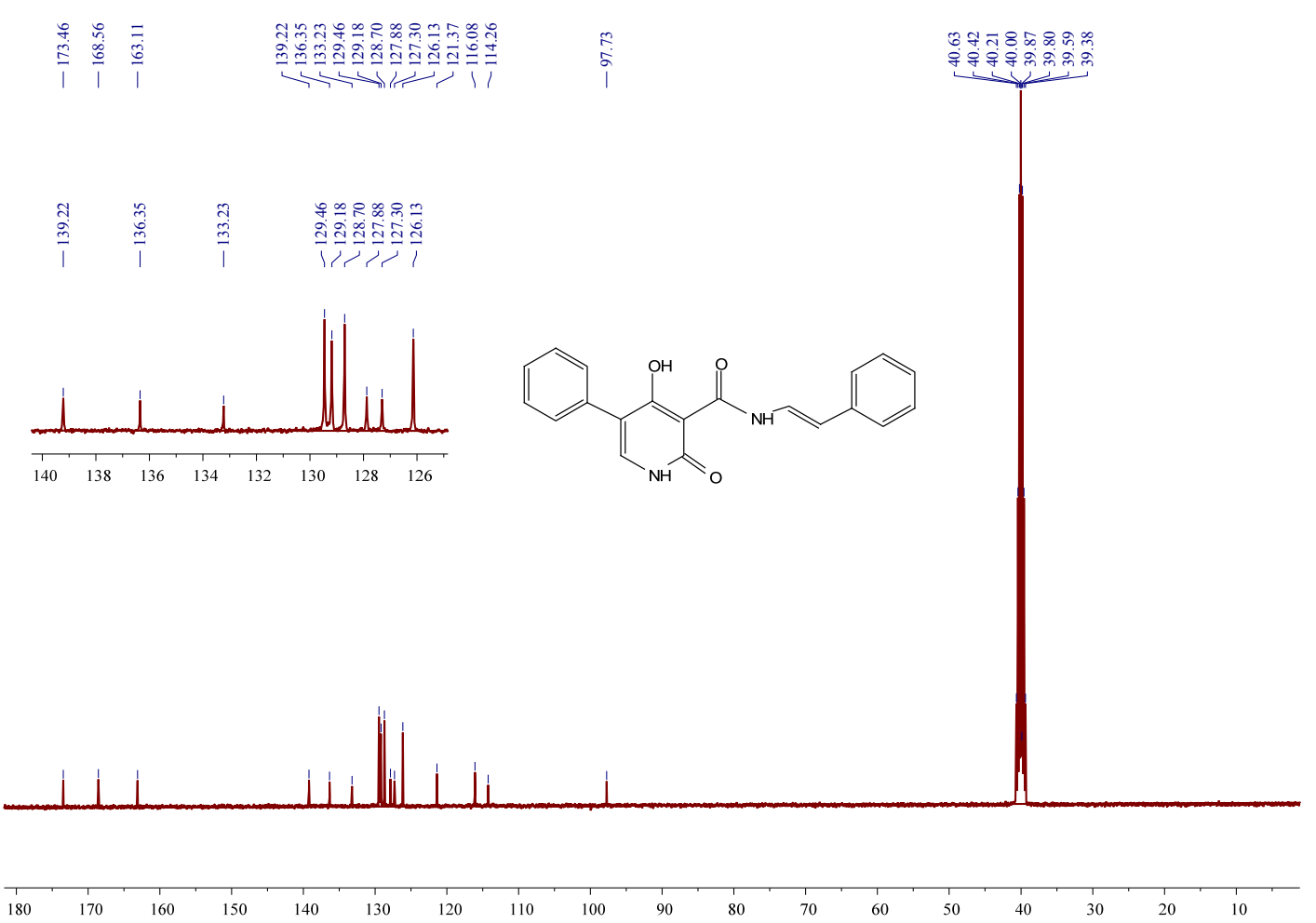

(E)-4-hydroxy-5-(4-methoxyphenyl)-N-(4-methoxystyryl)-2-oxo-1,2-dihydropyridine-3-carboxamide (6b)
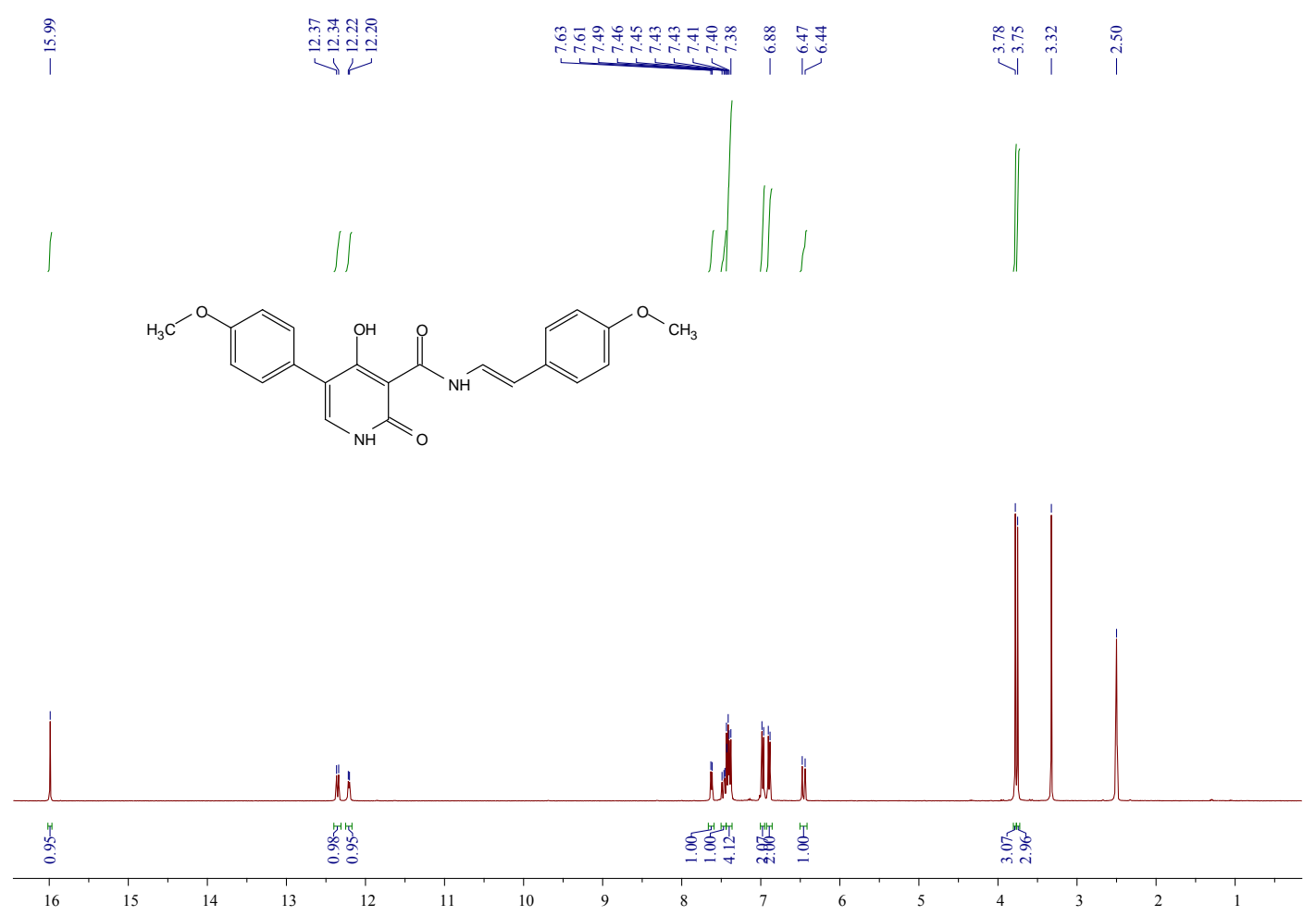


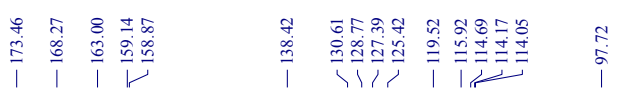

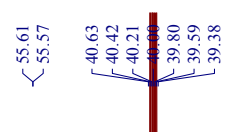

(N)
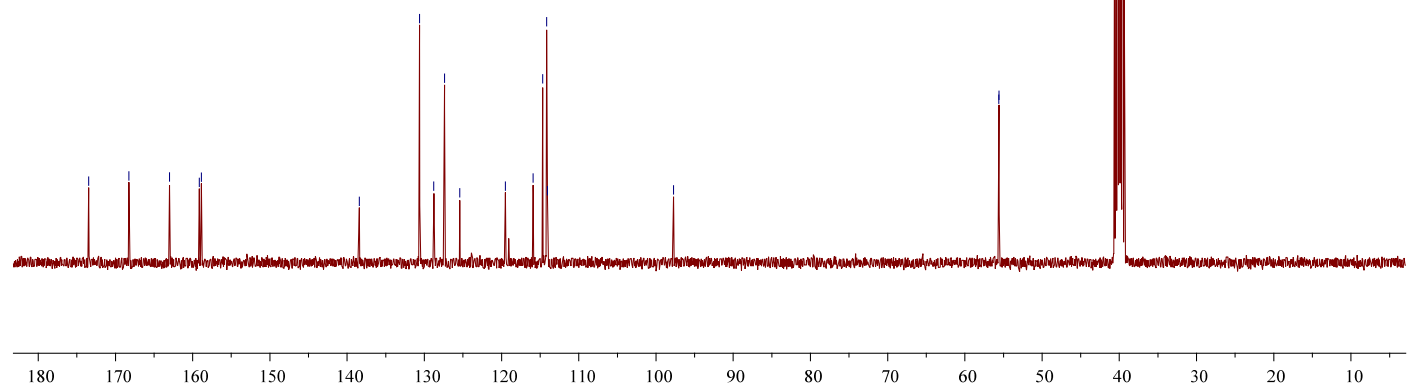

(E)-5-(4-chlorophenyl)-N-(4-chlorostyryl)-4-hydroxy-2-oxo-1,2-dihydropyridine-3-carboxamide (6c)

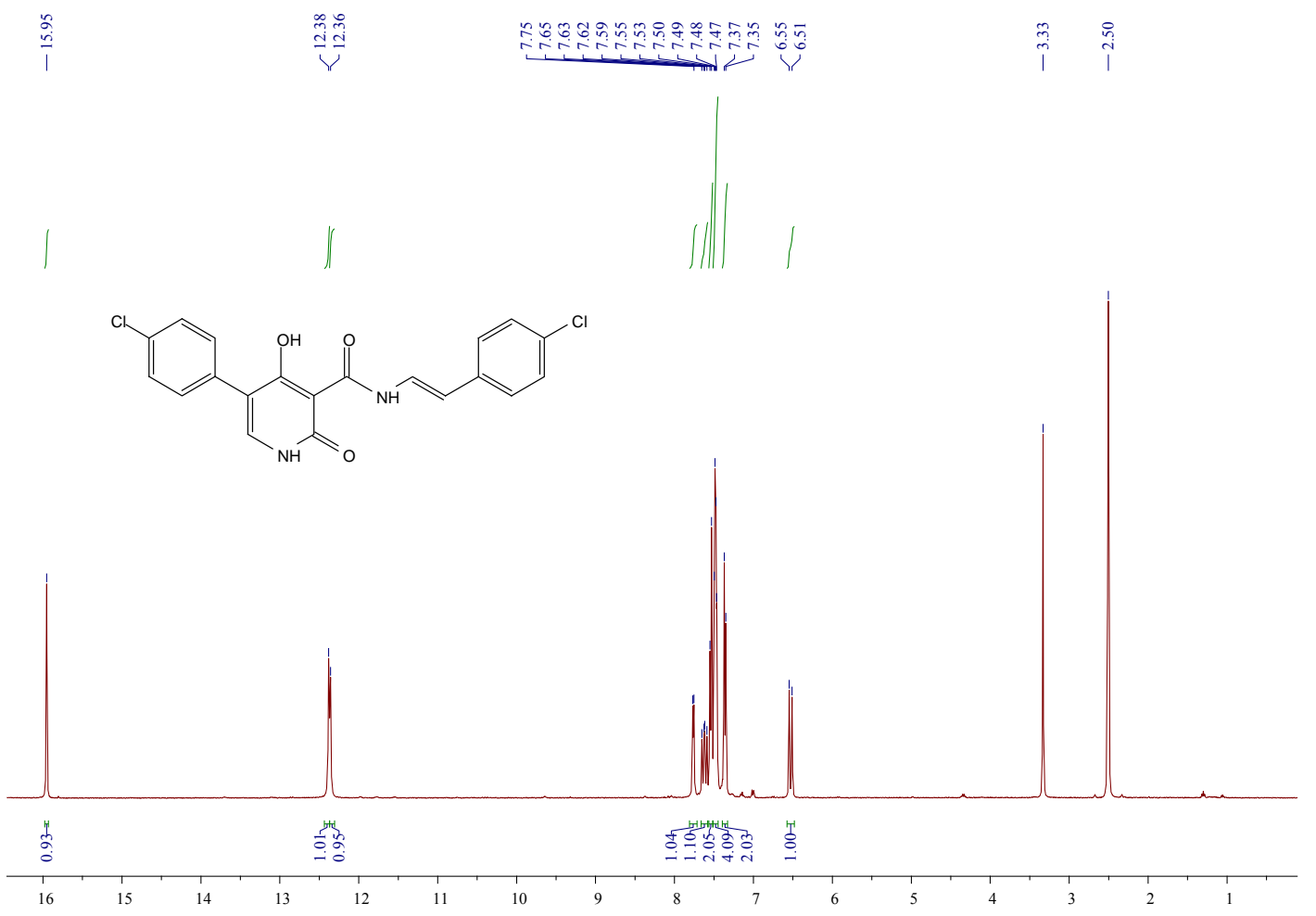




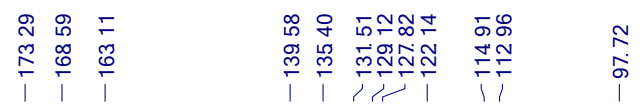

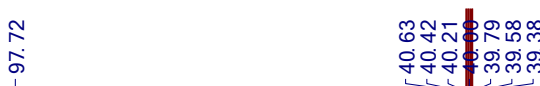

(c)

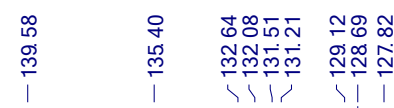

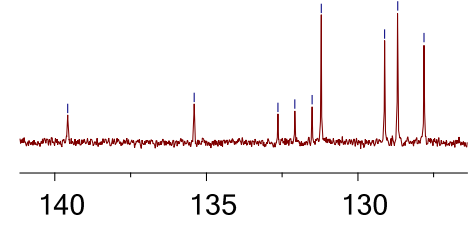

$\begin{array}{lllllllllllllllll}170 & 160 & 150 & 140 & 130 & 120 & 110 & 100 & 90 & 80 & 70 & 60 & 50 & 40 & 30 & 20 & 10\end{array}$

(E)-4-hydroxy-5-(4-nitrophenyl)-N-(4-nitrostyryl)-2-oxo-1,2-dihydropyridine-3-carboxamide (6d)

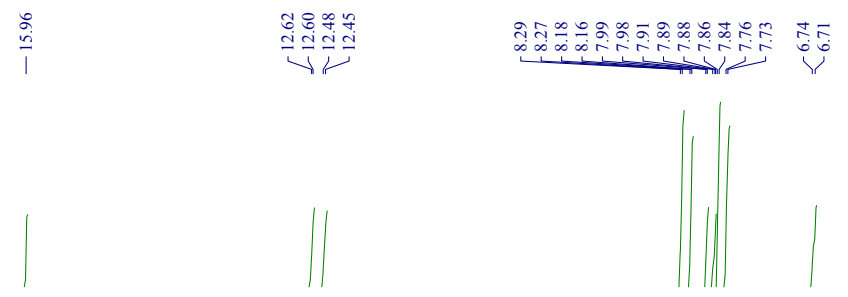<smiles>O=C(N/C=C/c1ccc([N+](=O)[O-])cc1)c1c(O)c(-c2ccc([N+](=O)[O-])cc2)c[nH]c1=O</smiles>

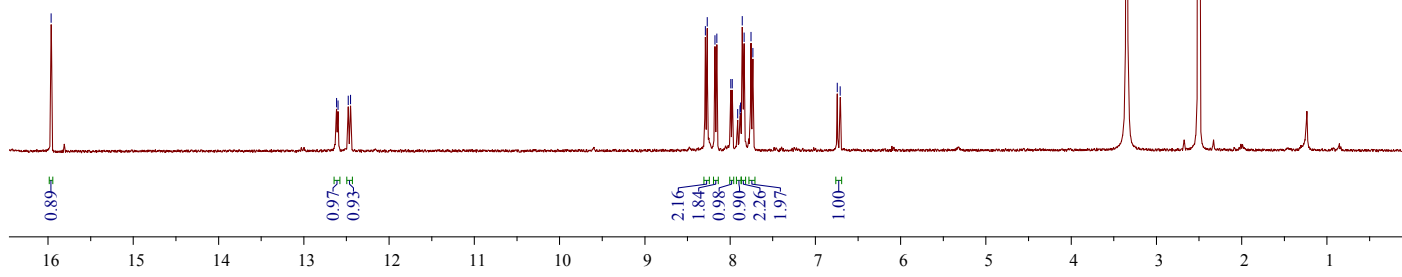




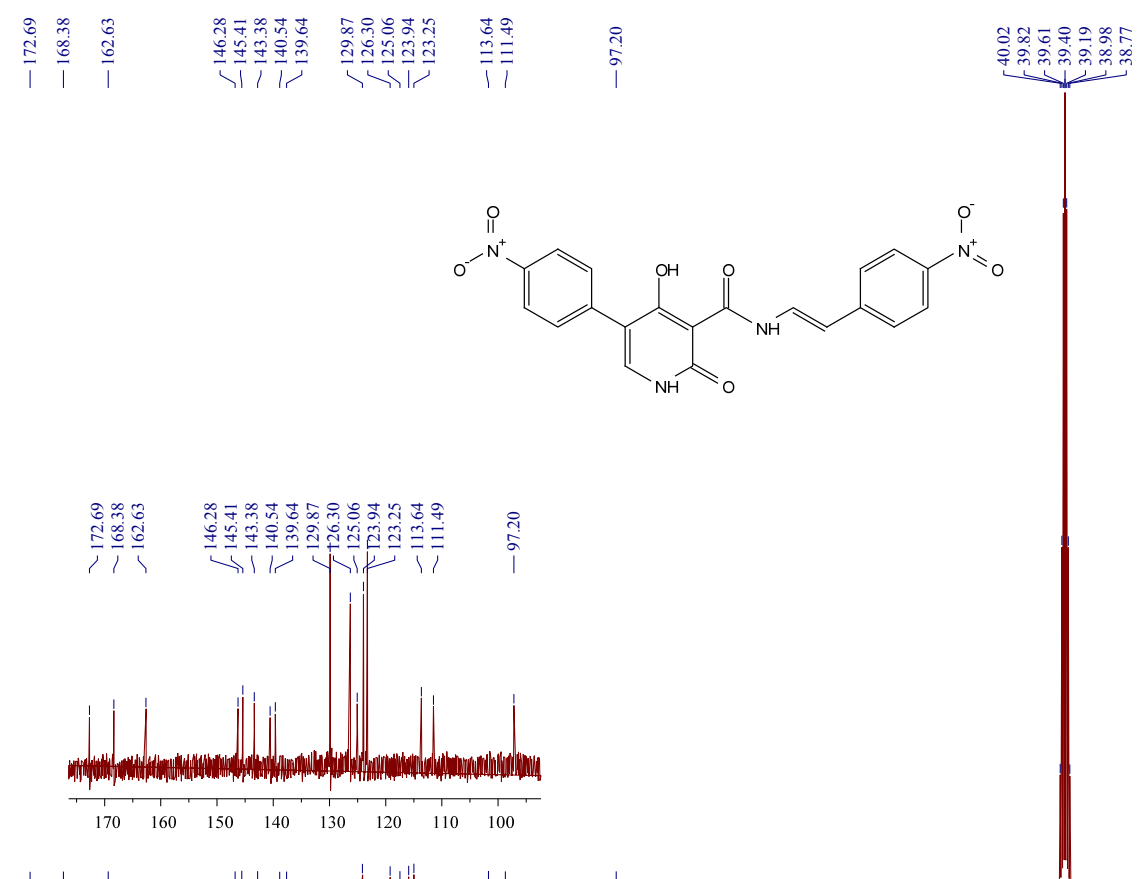

\begin{tabular}{|c|c|}
\hline 180 & 17 \\
\hline
\end{tabular}

${ }^{1} \mathrm{H}$ NMR and ${ }^{13} \mathrm{C}$ NMR spectra of Ethyl 4-hydroxy-2-oxo-1,2-dihydropyridine-

\section{3-carboxylates}

Ethyl 4-hydroxy-2-oxo-5-phenyl-1,2-dihydropyridine-3-carboxylate (7a)

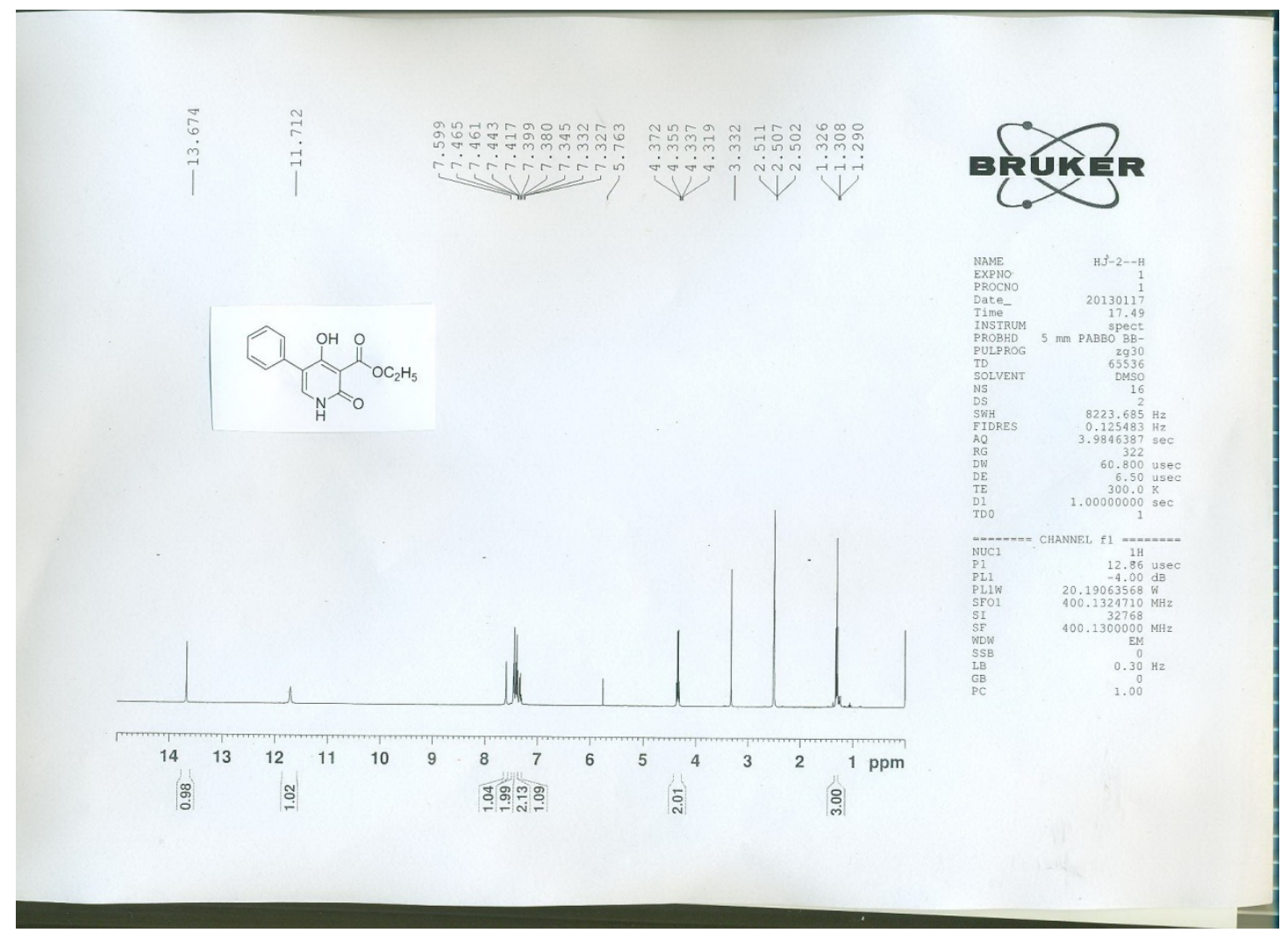




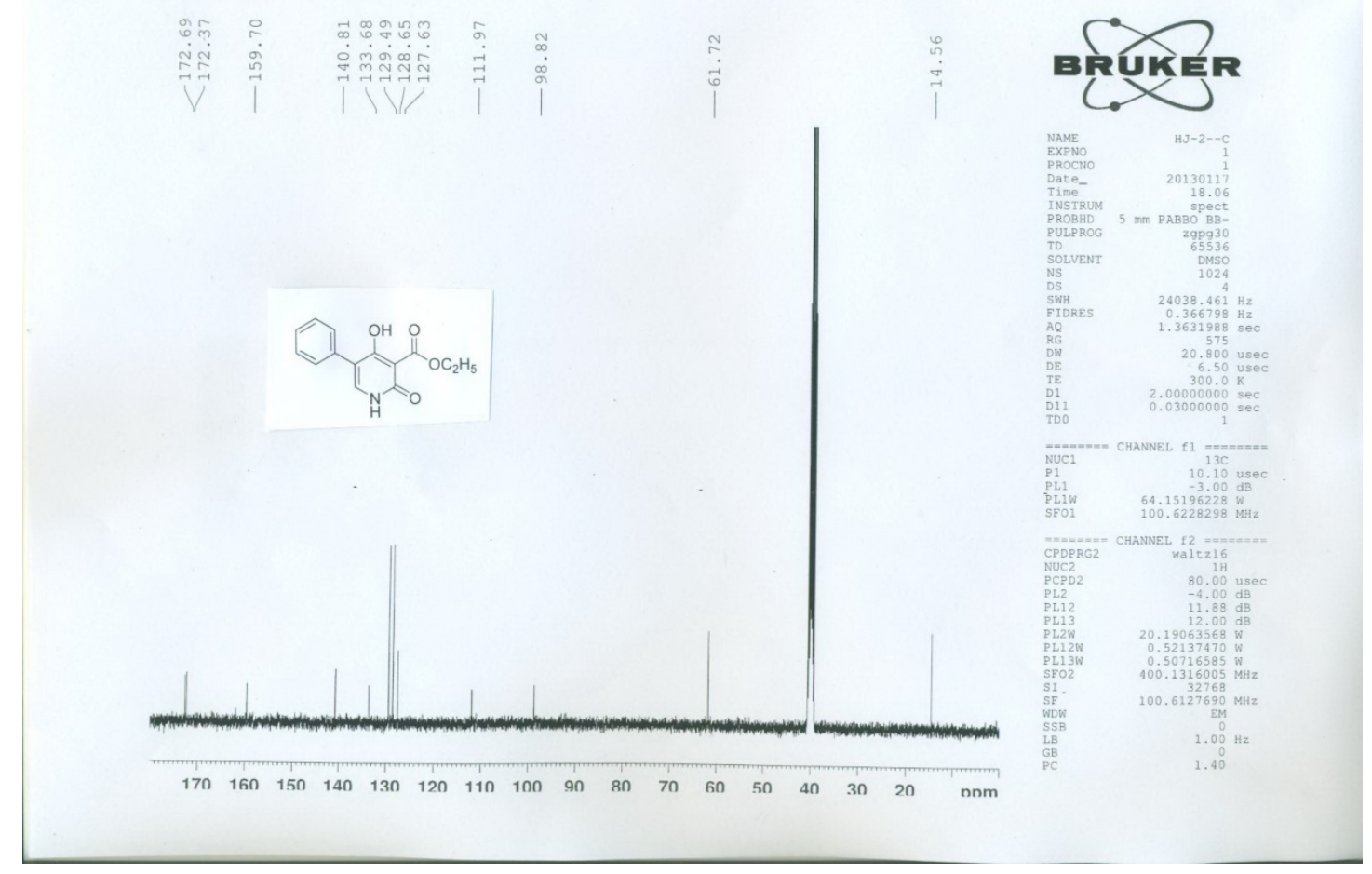

ethyl 4-hydroxy-5-(4-methoxyphenyl)-2-oxo-1,2-dihydropyridine-3-carboxylate (7b)
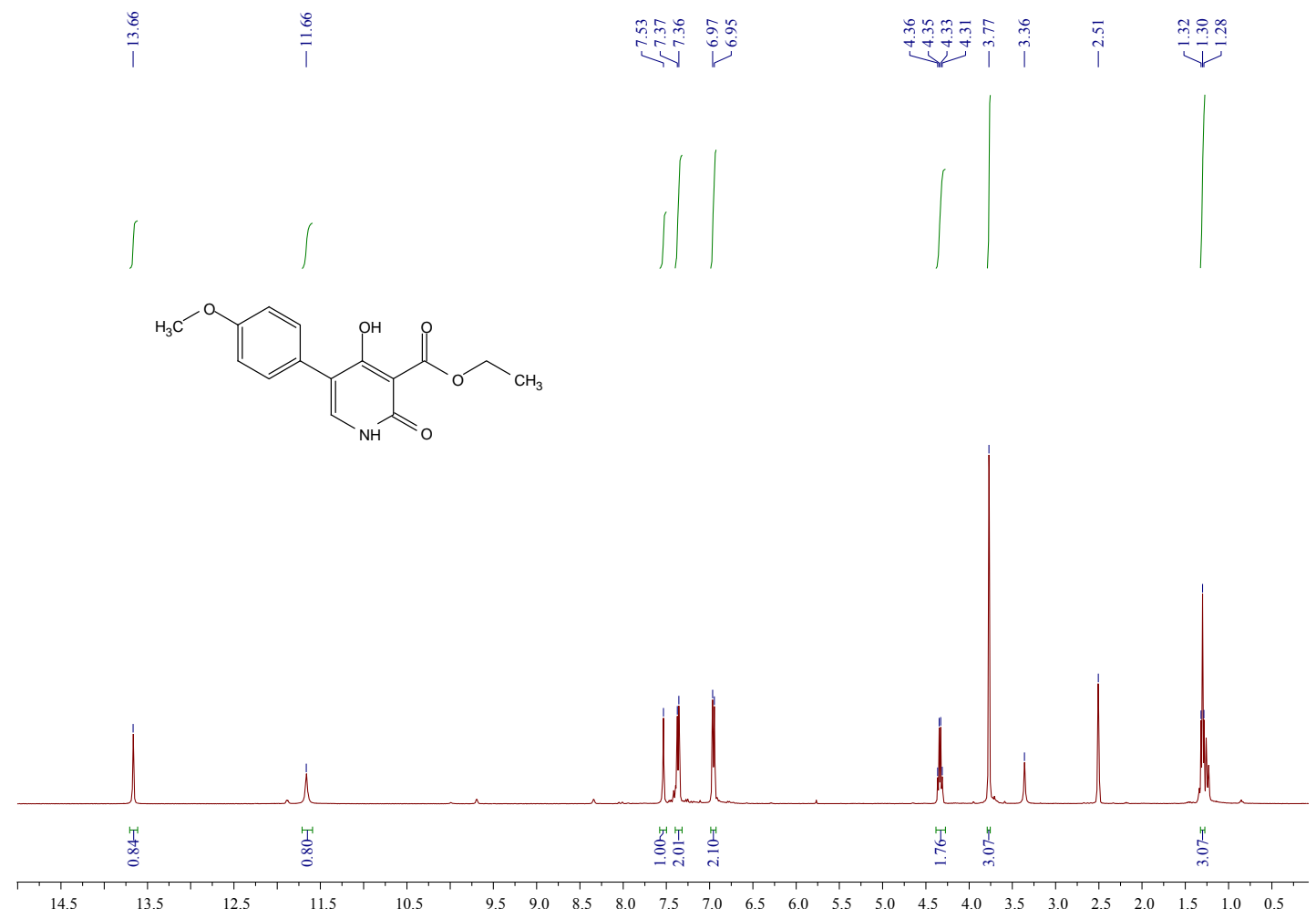

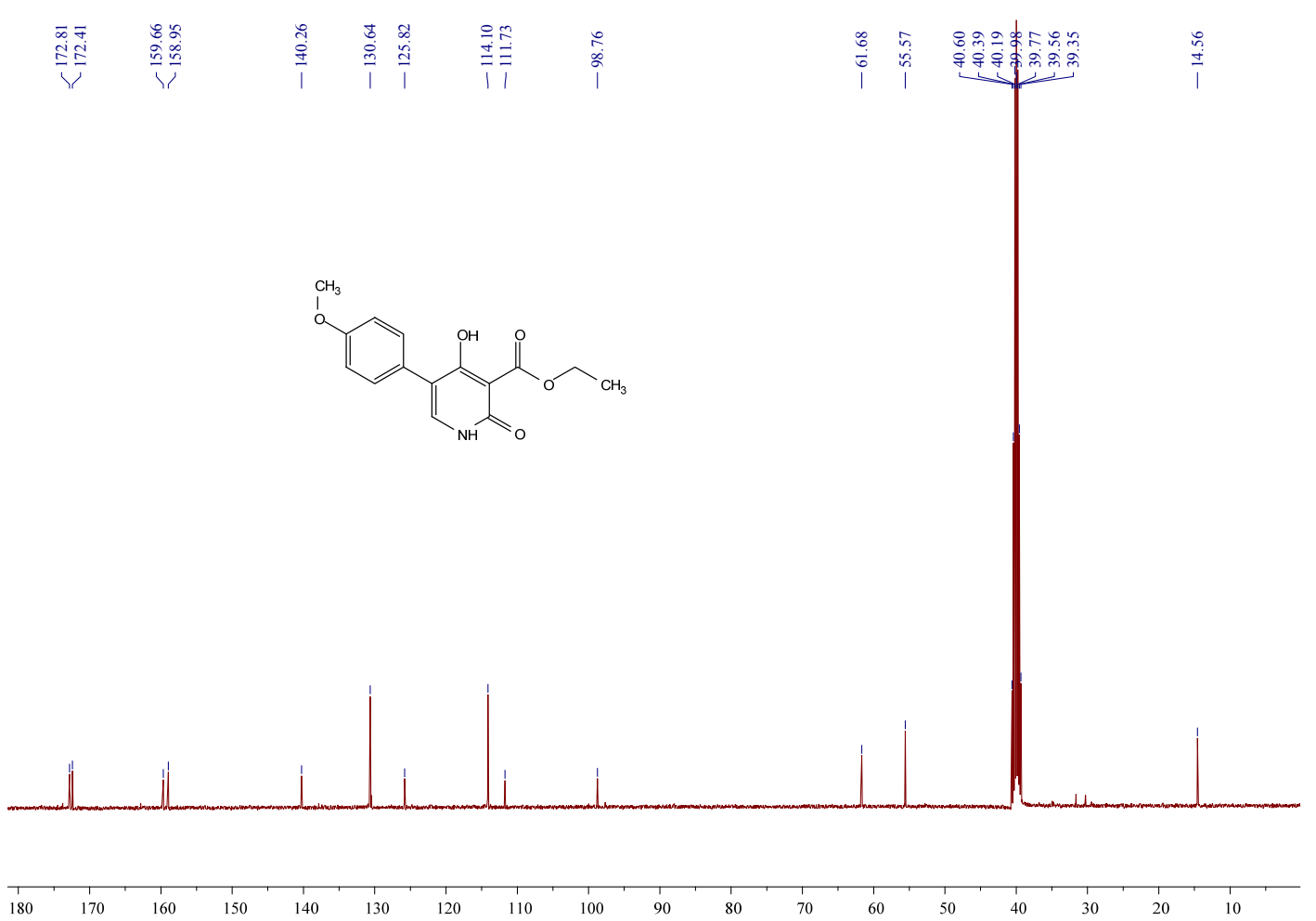

Ethyl 4-hydroxy-2-oxo-5-4-chlorophenyl-1,2-dihydropyridine-3-carboxylate (7c).

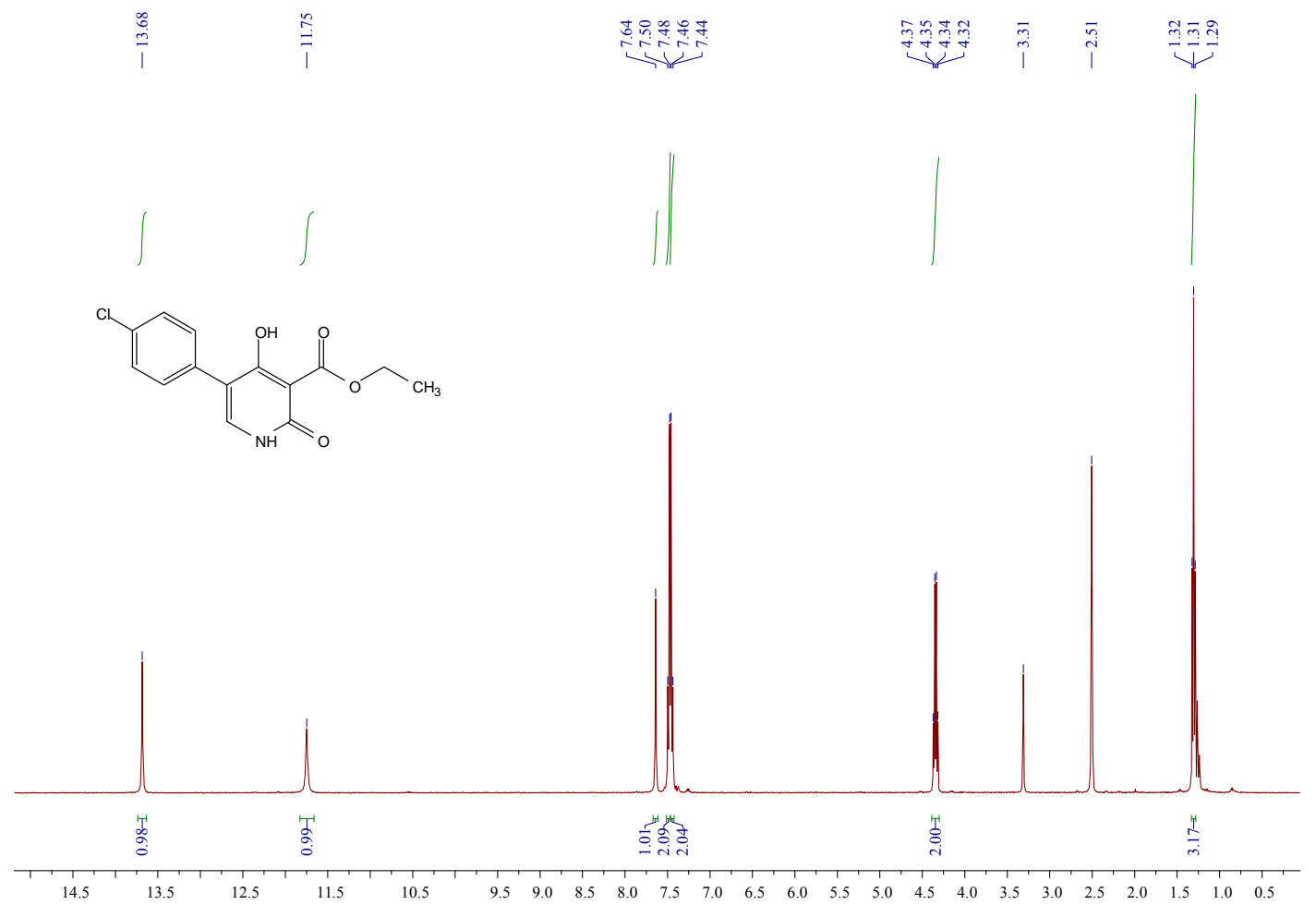




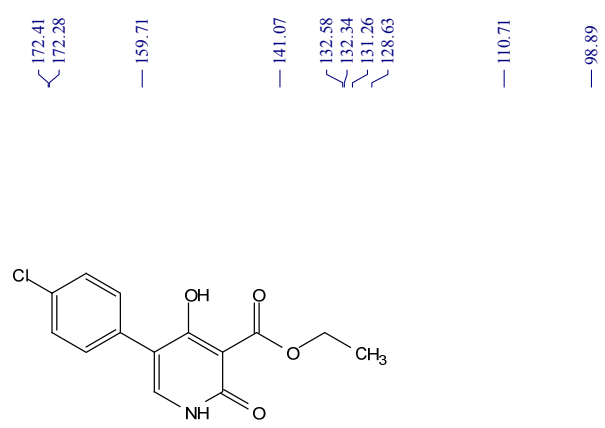

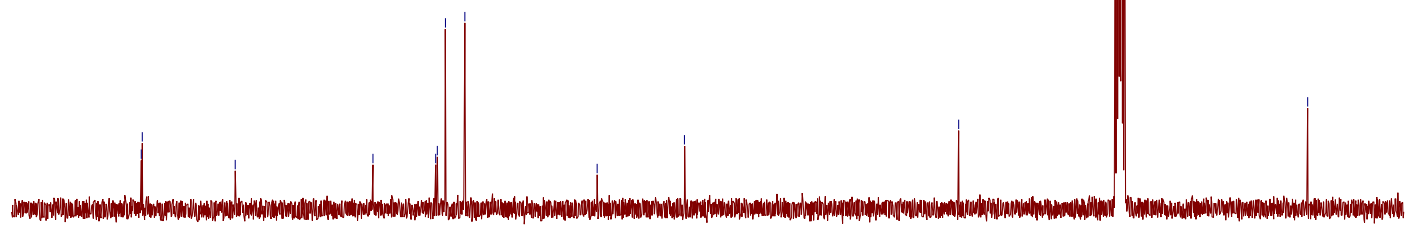

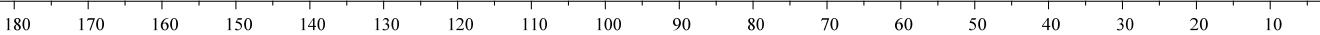

ethyl 4-hydroxy-5-(4-nitrophenyl)-2-oxo-1,2-dihydropyridine-3-carboxylate (7d)
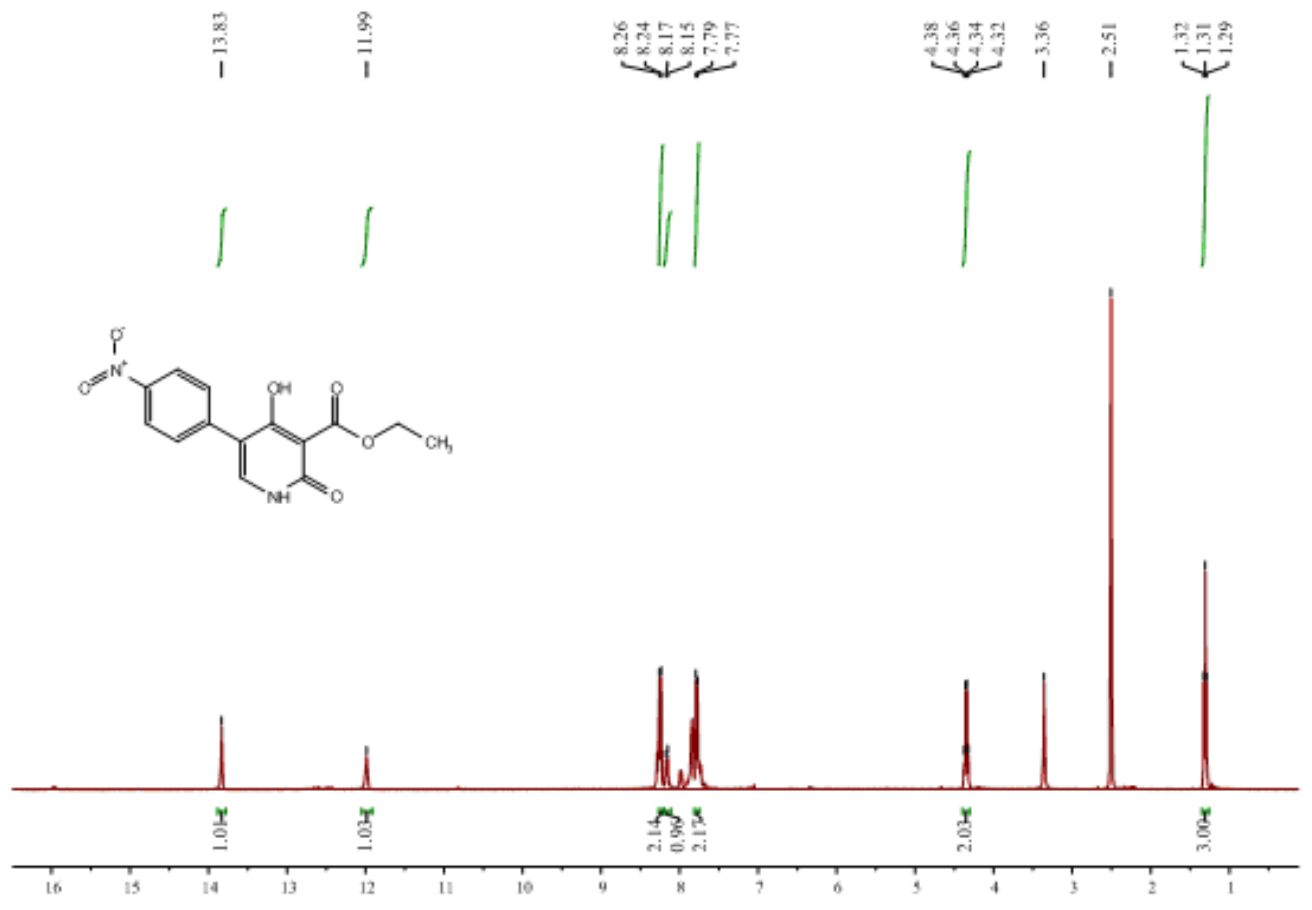

${ }^{1} \mathrm{H}$ NMR and ${ }^{13} \mathrm{C}$ NMR spectra of (E)-ethyl 2-((E)-styrylcarbamoyl)- 3-hydroxybut-2-enoates (E)-ethyl 3-hydroxy-2-(styrylcarbamoyl)but-2-enoate (12a) 


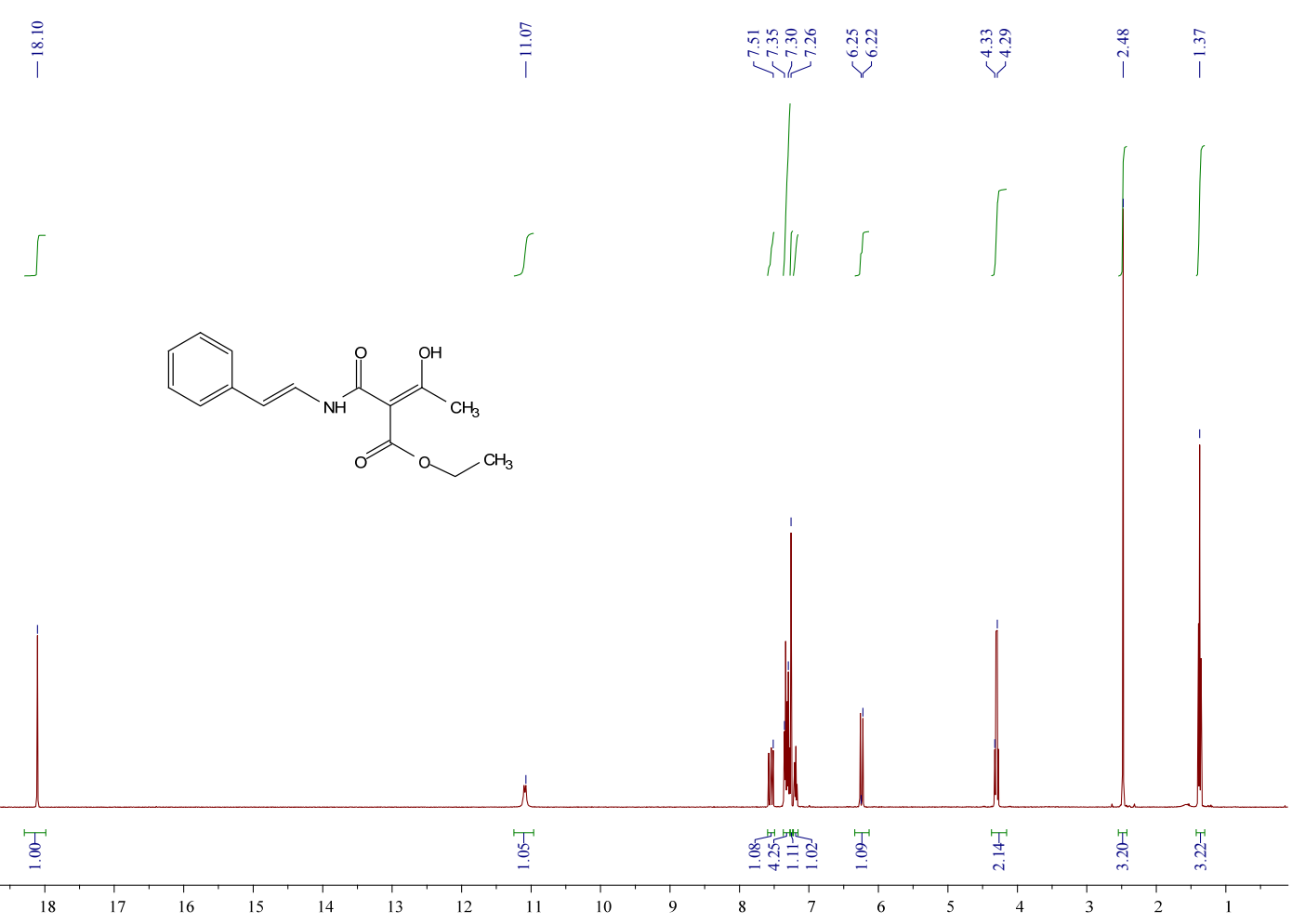

(E)-ethyl 3-hydroxy-2-(4-methoxystyrylcarbamoyl)but-2-enoate (12b)

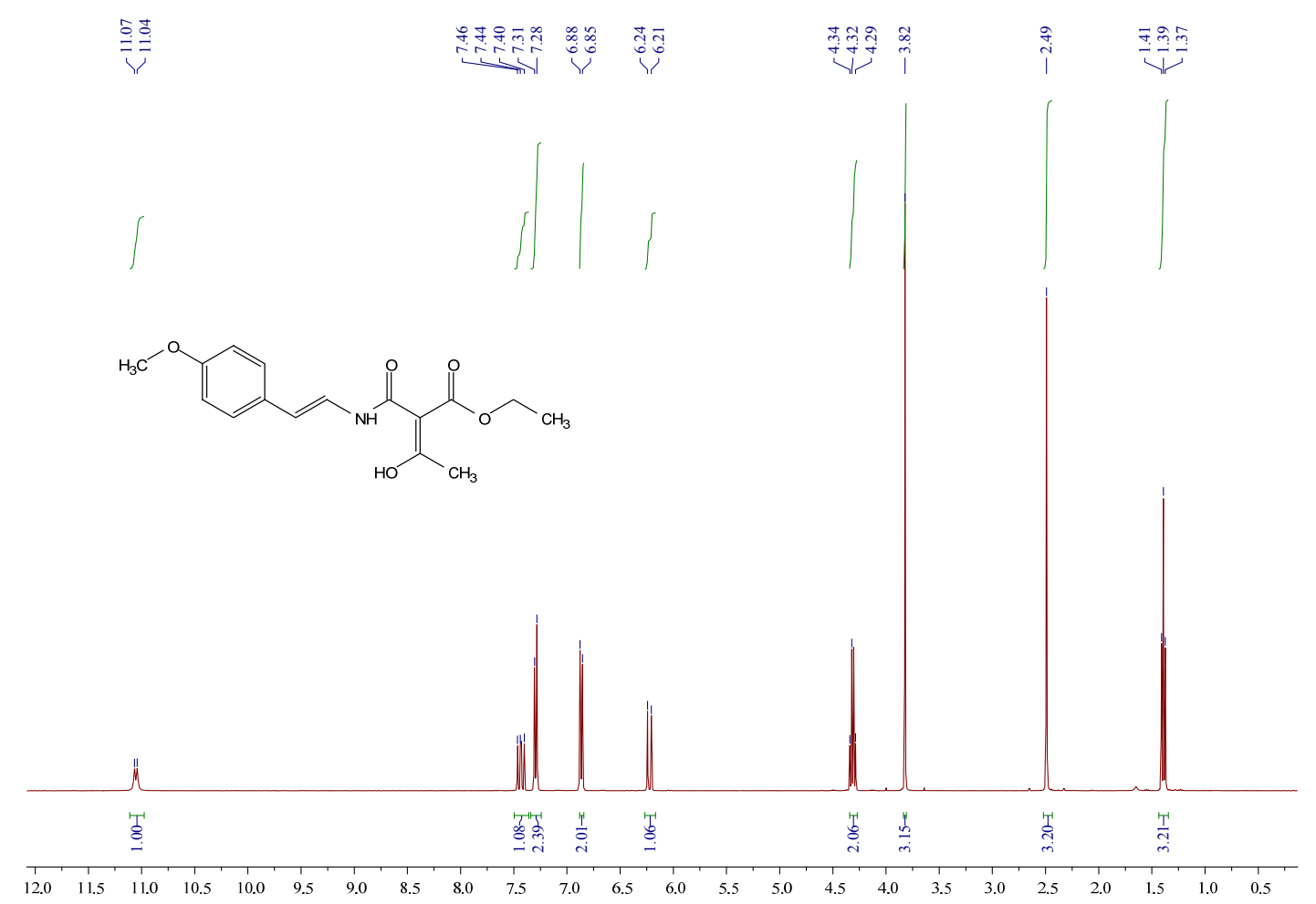

(E)-ethyl 2-(4-chlorostyrylcarbamoyl)-3-oxobutanoate (12c) 

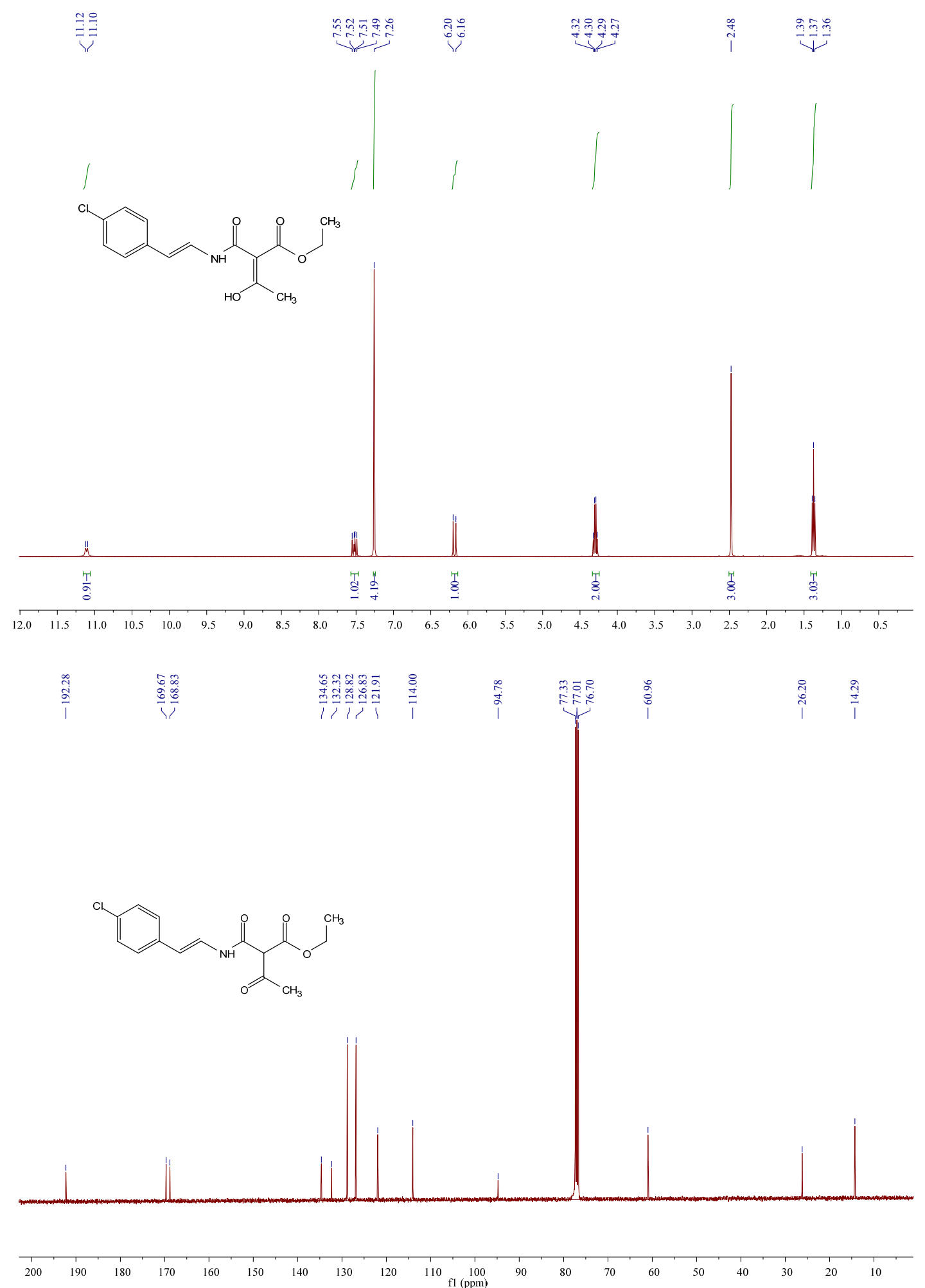

\section{${ }^{1} \mathrm{H}$ NMR and ${ }^{13} \mathrm{C}$ NMR spectra of 3-acetyl-4-hydroxypyridin-2(1H)-ones}

3-acetyl-4-hydroxy-5-phenylpyridin-2(1H)-one (13a) 


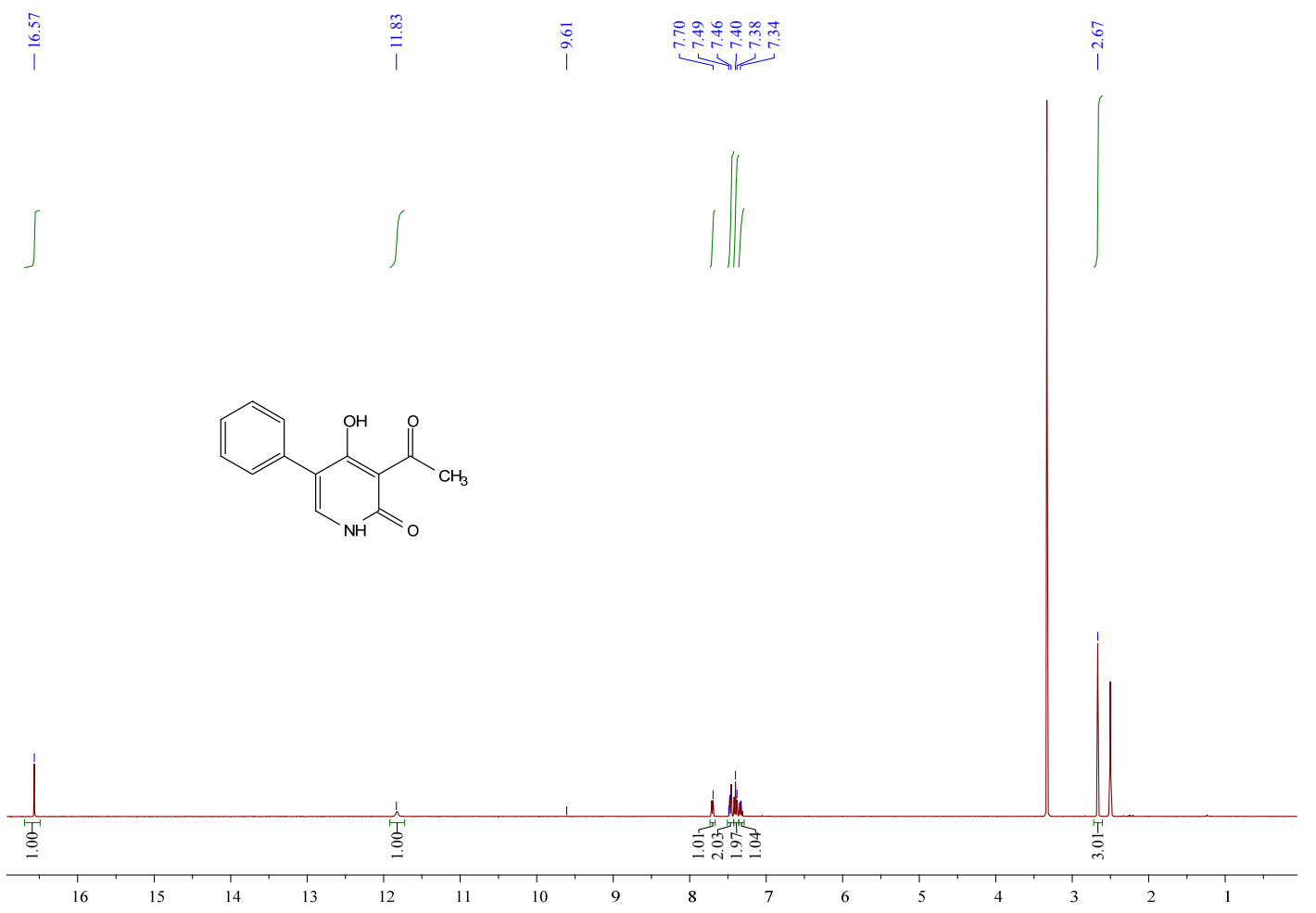

3-acetyl-4-hydroxy-5-(4-methoxyphenyl)pyridin-2(1H)-one (13b)

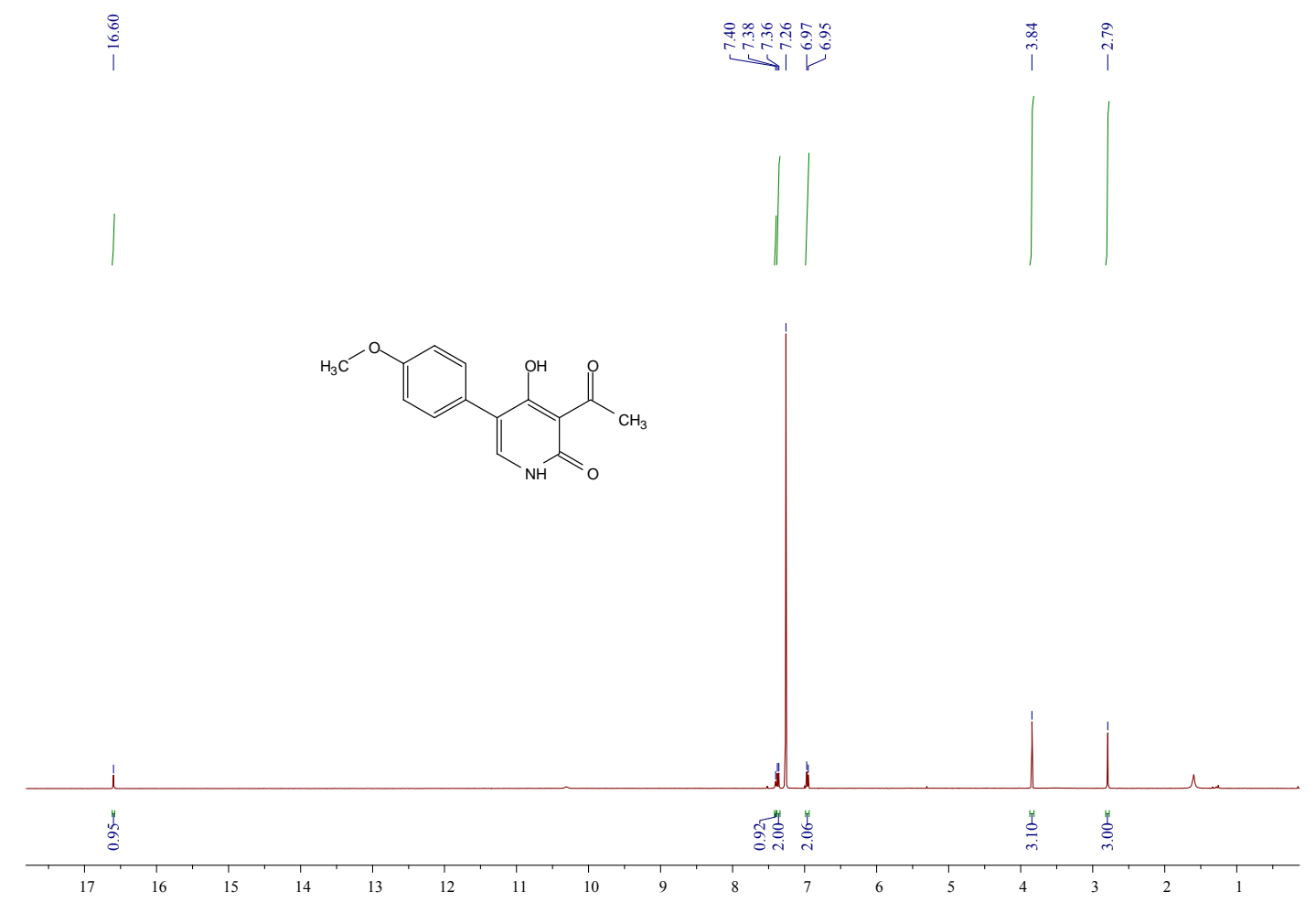

3-acetyl-5-(4-chlorophenyl)-4-hydroxypyridin-2(1H)-one (13c) 

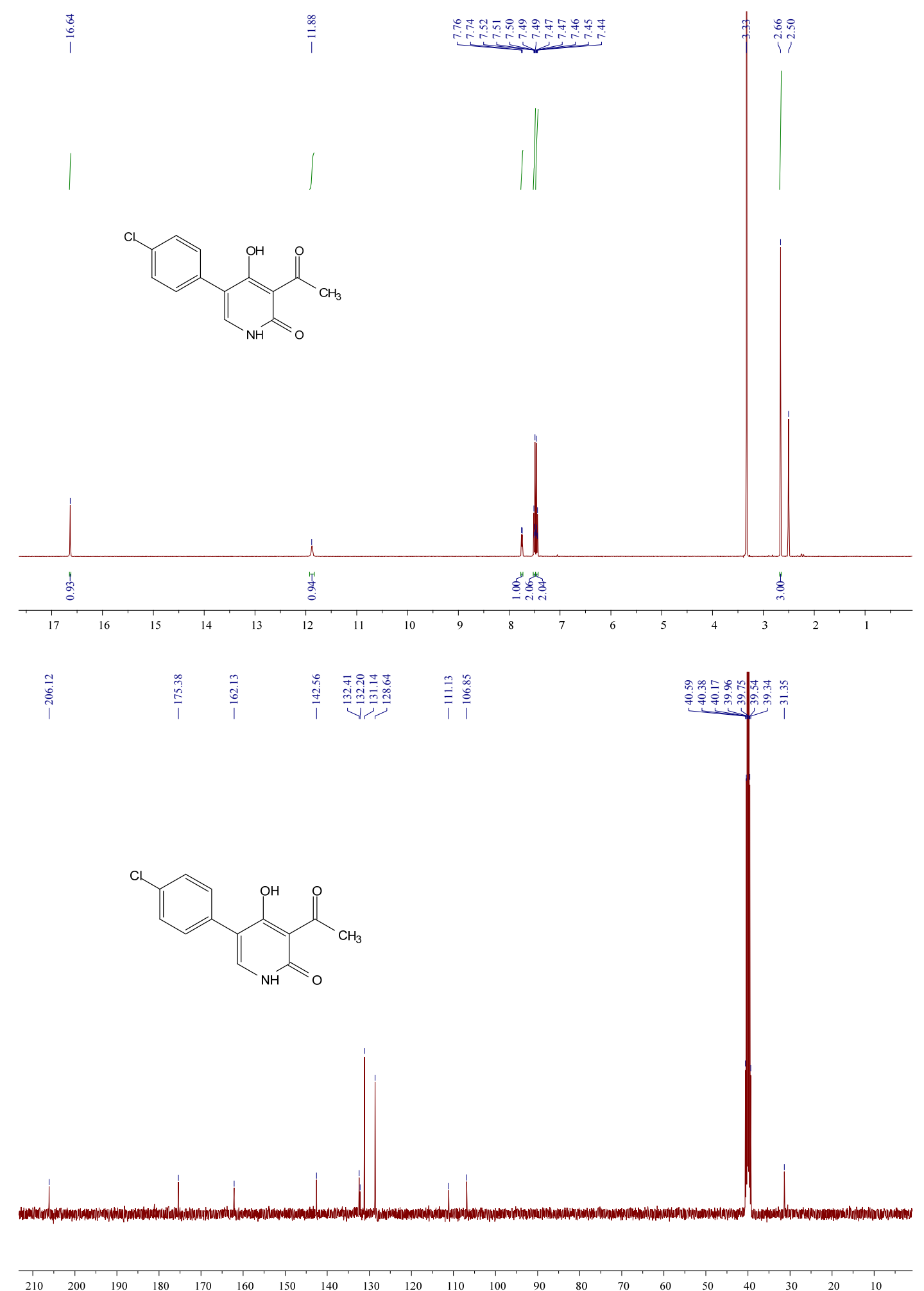

X-ray crystallographic data for compound ethyl 2-(((E)-4-methoxystyryl)carbamoyl) -3-hydroxybut-2-enoate 12b

Crystals of compound ethyl 2-(((E)-4-methoxystyryl)carbamoyl)-3-hydroxybut-2-enoate 12b 
suitable for X-ray analysis were obtained by slow evaporation of a solution of this compound in a mixture of ethyl acetate-petroleum ether solution (1:1). A single white crystal with dimensions $0.31 \times 0.27 \times 0.25 \mathrm{~mm}$ was chosen for X-ray diffraction study. The data were collected on a CAD-4 diffractometer equipped with graphite-monochromatic MoKa radiation $(\lambda=0.71073 \AA)$ using an $\omega$ scan mode at 293 (2) K. A view of the title structure is illustrated in Figure 1. A perspective view of the crystal packing in the unit cell is shown in Figure 2.

Data collection: SMART; cell refinement: SAINT; data reduction: SAINT; programs used to solve structure: SHELXS97; programs used to refine structure: SHELXL97; molecular graphics: SHELXTL; software used to prepare material for publication: SHELXTL. The structure was solved by direct methods with SHELXS-97. Non-hydrogen atoms were refined by full-matrix least-squares techniques on $F^{2}$ with anisotropic thermal parameters, using SHELXL-97 (Sheldrick, 2008). All $\mathrm{H}$ atoms were located in a difference Fourier map and refined with the riding model with $\operatorname{Uiso}(H)=1.2 \operatorname{Ueq}(C)$. The final full-matrix least-squares refinement gave $R_{1}=0.043$, $\mathrm{wR}_{2}=0.122$ for reflections with $\mathrm{I}>2 \sigma(\mathrm{I})$; the weighting scheme, $\omega=1 /\left[\sigma 2\left(F^{2}\right)+(0.0681 P)^{2}+0.143 \mathrm{P}\right]$, where $\mathrm{P}=\left(F_{\mathrm{O}}^{2}+2 F_{\mathrm{C}}^{2}\right) / 3$. Crystallographic data for the structure in this paper have been published in Acta Crystallographica, Section E: Structure Reports Online, 2012, E68, o798. This paper can be obtained free of charge via www.journals.iucr.org.

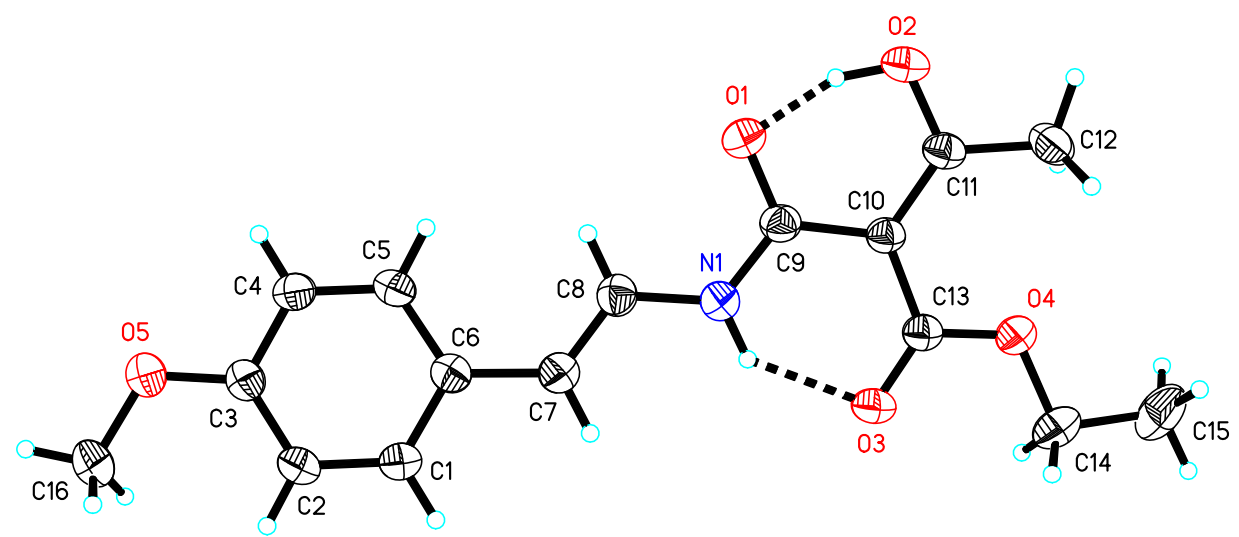

Figure 1 ORTEP structure of the compound12b, showing 50\% probability ellipsoids. 


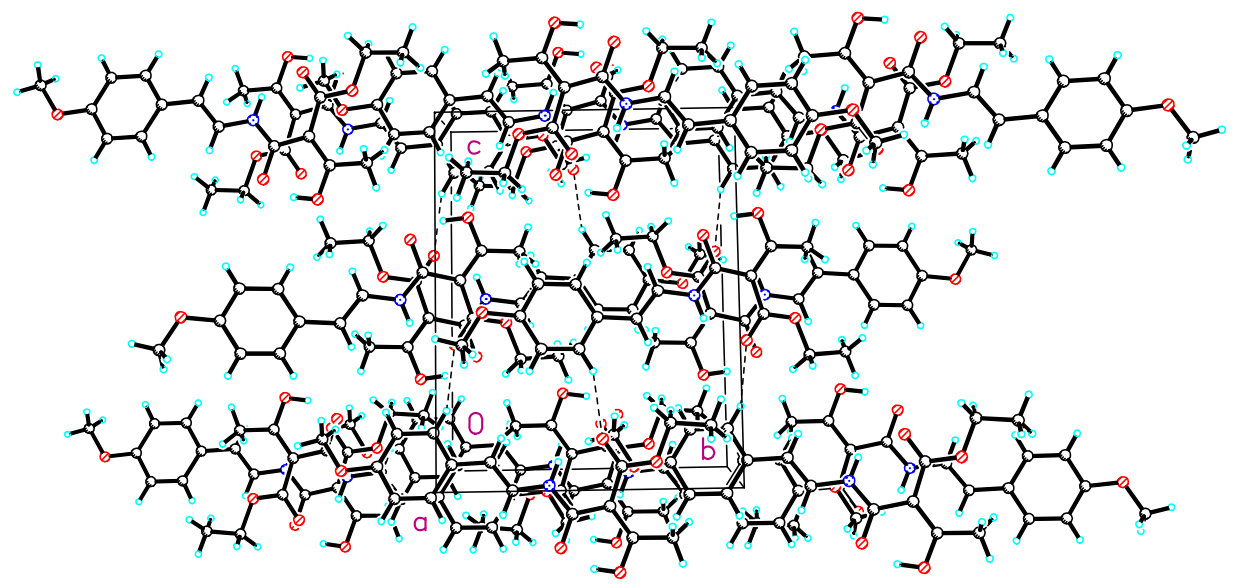

Figure 2 Packing view of the compound $\mathbf{1 2 b}$ viewed down the $a$ axial direction, with intermolecular $\mathrm{C}-\mathrm{H} \cdot$ • •

O hydrogen bonding shown as dashed lines. 